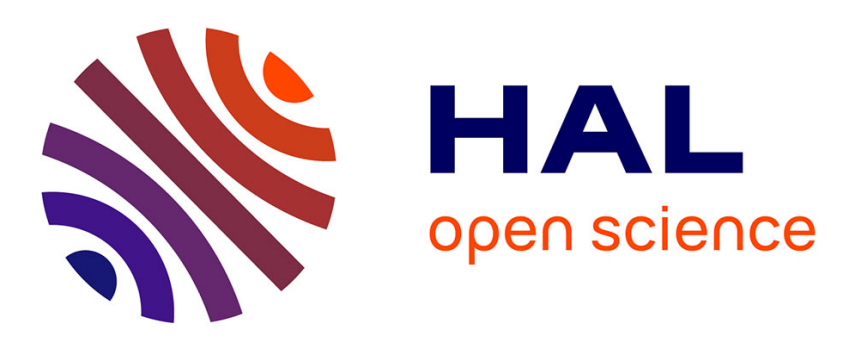

\title{
Light-induced deformation and instability of a liquid interface. I. Statics.
}

Régis Wunenburger, Alexis Casner, Jean-Pierre Delville

\section{To cite this version:}

Régis Wunenburger, Alexis Casner, Jean-Pierre Delville. Light-induced deformation and instability of a liquid interface. I. Statics.. Physical Review E: Statistical, Nonlinear, and Soft Matter Physics, 2006, 73 (3), pp.036314 (1-14). 10.1103/PhysRevE.73.036314 . hal-00389843

\section{HAL Id: hal-00389843 \\ https://hal.science/hal-00389843}

Submitted on 29 May 2009

HAL is a multi-disciplinary open access archive for the deposit and dissemination of scientific research documents, whether they are published or not. The documents may come from teaching and research institutions in France or abroad, or from public or private research centers.
L'archive ouverte pluridisciplinaire HAL, est destinée au dépôt et à la diffusion de documents scientifiques de niveau recherche, publiés ou non, émanant des établissements d'enseignement et de recherche français ou étrangers, des laboratoires publics ou privés. 


\title{
Light induced deformation and instability of a liquid interface- I. Statics.
}

\author{
Régis Wunenburger, ${ }^{*}$ Alexis Casner, ${ }^{\dagger}$ and Jean-Pierre Delville ${ }^{\ddagger}$ \\ Centre de Physique Moléculaire Optique et Hertzienne, Université Bordeaux I, \\ 351 cours de la Libération, 33405 Talence Cedex, France
}

\begin{abstract}
We study in details the deformations of a liquid-liquid interface induced by the electromagnetic radiation pressure of a focused cw laser beam. Using a simple linear model of static equilibrium of the interface under the effect of radiation pressure, buoyancy and Laplace pressure, we explain the observed hump height variations for any value of the optical Bond number $B o=\left(\omega_{0} / \ell_{c}\right)^{2}\left(\ell_{c}\right.$ is the capillary length and $\omega_{0}$ is the waist of the beam) in the regime of weak deformations, and show that the deformations are independent of the direction of propagation of the laser. Increasing the beam power, we observe an instability of the interface leading to the formation of a long jet when the laser propagates from the more refringent phase to the less refringent one. We propose that the total internal reflection of the incident light on the highly deformed interface could be at the origin of this instability. Using a nonlinear model of static equilibrium of the interface taking account of the angular dependance of radiation pressure, we explain the observed beam power threshold of the instability as well as the shape of the interface deformations observed at large waists just below the instability onset. According to this model, the instability should occur when the interface slope reaches the angle of total reflection $\theta_{T R}$. We find that the maximum incidence angle along the interface $\theta_{\mathrm{i} \text { max }}$ just below the instability threshold is significantly smaller than $\theta_{T R}$, and that our nonlinear model does not present any instability up to $\theta_{\mathrm{i} \max }=\theta_{T R}$, making the proposed instability mechanism qualitatively rough although quantitatively accurate. We finally discuss possible additional effects that could explain the instability.
\end{abstract}

PACS numbers: 47.20.Ma 42.50.Vk 42.25.Gy 68.05.-n

\footnotetext{
*Electronic-mail: r.wunenburger@cpmoh.u-bordeaux1.fr

†Present address: Département de Conception et de Réalisation d’Expériences, CEA/DAM Ile-de-France, BP 12, F-91680 Bruyères-le-Châtel, France; Electronic-mail: alexis.casner@cea.fr
} 
${ }_{\ddagger}^{\ddagger}$ Electronic-mail: jp.delville@cpmoh.u-bordeaux1.fr 


\section{INTRODUCTION}

Since the Maxwell's calculation of the sunlight radiation pressure on a mirror [1], the force exerted by light on surfaces is a topic of intense discussions [2-4]. Maxwell predicted that a light beam of intensity $I$ impinging normally upon a surface of reflectivity $R$ exerts a pressure $\Pi_{r}$ analogous to that of a beam of material particles. In 1861, he derived the following formula: $\Pi_{r}=(1+R) I / c$. The first attempt at an experimental proof of the existence of the radiation pressure $\Pi_{r}$ on a mirror in vacuum was performed by Lebedev [5] in 1901. The experiments was further improved by Nichols and Hull [6] in 1903. Both calculated and observed surface forces were proved to act inwards to the reflecting mirror. In 1905, Poynting [7] extended the treatment of the radiation pressure to the case of light refraction. By considering light incident from vacuum on the surface of a transparent dielectric, he predicted an outward force, normal to the surface, irrespective of the angle of incidence. This was in agreement with previous calculations from Thomson [8]. An outward normal force has indeed been experimentally demonstrated by Ashkin [9] when a laser beam crosses the water free surface. However, these observations revealed, in fact, a still continuing controversy for the expression of the photon momentum in dielectric media; depending on the expression of the momentum density of the electromagnetic field inside a dielectric, either the Abraham or the Minkowski form, the surface force is predicted to act inwards or outwards. We refer the reader interested in this conflict on radiation pressure theory to nice reviews and articles as those written by Brevik [10], Loudon [11], Obukhov [12], or Mansuripur [3]. Despite Ashkin's observations, it was concluded that the direction of the fluid interface deformation could not be considered as a sufficient proof to definitely answer the controversy because laser waves also give birth to radial forces due to their Gaussian intensity profile $[11,13]$. Conversely, contrary to the theoretical point of view, experiments converge towards a unified picture. Indeed, since the seminal work of Ashkin [9], all the investigations performed with lasers on fluid interfaces have invariably retrieved the behavior predicted by Poynting, i.e. the emergence of an outward surface force [14-17]. The generalization to interfaces between two dielectrics leads to surface forces directed towards the medium of lowest index of refraction $[18,19]$.

As illustrated in the following, the optical surface force is proportional to the refractive index contrast between the media on both sides of the interface. Consequently, the am- 
plitude of the expected optical forcing is intrinsically weak in any case. Then, since the radiation pressure has to compete with the surface tension (Laplace pressure) and buoyancy (hydrostatic pressure), the resulting interface deformation is also very weak in general. The effect of the surface force is thus difficult to observe. Typically, the height of the deformation induced at the water free surface by a focused continuous Argon ion laser of a few watts reaches a ten of nanometers [16]. Consequently, most of the experiments were performed with laser pulses to compensate the weakness of the index contrast by the irradiance. There exist nevertheless three major drawbacks. (i) With pulse excitation the control over the spatial and temporal profiles of the wave is not always sufficiently accurate for reproducibility in measurements. (ii) Stationary behaviors are never observed. (iii) Intense irradiance often sets off secondary disturbing bulk couplings such as optical nonlinearities (self-focusing and filamentation) and/or excess heating [20] (thermal expansion and boiling). Bulk heating by lasers can as well directly couple with liquid interfaces and significantly deform them by thermocapillary flows [21]. On the other hand, due to its weakness, the induced surface force is often investigated indirectly from the lensing associated to the interface deformation $[9,16,17]$ or by interferometric holography [14]. This weakness also suggests why radiation pressure effects received much less attention than optical trapping and tweezing [22]. The notoriety of optical tweezers even leads to situations where surface properties were indirectly probed by optical actuation of beam trapped beads in contact with the interface [23] or "glued" to it [24]. However, the emergence of soft materials in the eighties removed the laser radiation pressure from its confinement to the optical physics area, and brought a sort of second birth to optical surface forces. Indeed, as surfactants and membranes are often characterized by low surface tension, it became possible to directly deform soft interfaces at reasonably low irradiance without the help of a beam-trapped bead. This property allows for quantitative measurements of ultra-low surface tension in a contactless manner [19]. Moreover, since optical dynamometry is generally limited to large vesicles, radiation pressure was also used to quantitatively determine the elasticity of small vesicles [25] and cells [26, 27]. As the bending of these soft interfaces was still weak, all these experiments, and others [18], essentially investigated the linear regime of deformation. If now this regime starts to be well documented, our understanding of non-linear behaviors in deformations appearing at much larger laser illuminations, when reachable, is still at a very early stage. Impressive pictures of water droplets shape distortions driven by laser pulses were already presented more than 
fifteen years ago by Zhang and Chang [15]. In their experiments, large deformations at the front and the rear of a water droplet intercepted by the beam were observed with eventual free-surface disruption on the exit face under even higher illumination. Even if significant success has been achieved in the theoretical description of the weakly non-linear regime of deformation [28, 29], such a scheme, based on linear wave theory, cannot explain giant deformations and the subsequent droplet disruption.

Using very soft transparent liquid interfaces, the aim of the present research is, namely, to give a general description of these optical radiation pressure effects. We experimentally investigate the shape and the amplitude of stationary laser-induced interface deformations from the linear to the highly nonlinear regime until the onset of interface instability. Results are compared to predictions from existing models. Moreover, by investigating the curvature of interface deformations very close to the instability onset, we discuss the validity of the instability mechanism previously suggested [30]. To explore the underlined physics, we used near-critical liquid-liquid interfaces. As surface tension vanishes close to a critical point, this near-criticality offers invaluable conditions to investigate the full range in interface deformation at steady state under continuous wave laser excitation, i.e. in conditions that are particularly favorable for quantitative investigations. By varying the interface softness with a temperature scanning and changing the size of the exciting beam, it becomes indeed possible to give a sort of universal point of view on laser-induced interface deformation. The related dynamics and the corresponding time scales close and far from instability onset are discussed in details in a companion article [31].

We first present in Sec. II the characteristic features of the electromagnetic radiation pressure and of the experimental setup. Sec. III is devoted to the theoretical and experimental results concerning the static interface deformations of small amplitude (so-called "linear regime") induced by the radiation pressure. In particular, we show that the height and the shape of small amplitude deformations do not depend on the direction of propagation of the beam ("up-down" invariance). At large enough beam power, this up-down invariance breaks down, and the interface shapes vary with the direction of propagation of the laser beam. In Sec. IV, we study the interface instability which occurs when the incident laser beam exceeds a given power, leading to the formation of a long jet. We propose a simple mechanism of instability that accurately predicts the values of the beam power at which the instability develops. This model is based on the hypothesis that instability occurs 
when the maximum of the angle of incidence along the deformed interface reaches the angle of total reflection. This leads to a focusing of the incident electromagnetic energy towards the tip of the deformation and consequently to a huge increase of the radiation pressure acting on it. In order to check the validity of this instability mechanism, we measure the angle of incidence along the deformed interface at the instability threshold. We show that its maximum is noticeably smaller than the angle of total reflection, implying that only a small amount of the incident electromagnetic energy is reflected towards the deformation tip at the instability threshold. In Sec. V, we finally show that a simple nonlinear numerical model of the interface deformation describes satisfactorily the observed deformations just below the instability threshold, although it does not present any numerical instability. Using this numerical computation, we explain why the model of total internal reflection, although crude, accurately predicts the beam power threshold at which the instability occurs. Finally, we conclude in Sec. VI.

\section{PRINCIPLES OF THE EXPERIMENT}

In this section, we first present the main characteristic features of the electromagnetic radiation pressure at the interface between two dielectric fluids. The fact that radiation pressure is usually very weak justifies our choice of a very soft liquid interface, whose properties are next shortly presented. We finally describe the experimental setup used for deforming and visualizing deformed liquid interfaces.

\section{A. Electromagnetic radiation pressure at an interface between two dielectric me-} dia

To figure out a simple and intuitive picture of the electromagnetic radiation pressure, let us consider the flat interface between two dielectric, non-magnetic media labelled 1 and 2, of uniform refractive index $n_{1}$ and $n_{2}\left(n_{1}<n_{2}\right)$. We assume this interface to be irradiated at normal incidence by a monochromatic light beam (frequency $\nu$, direction of propagation along unit vector $\mathbf{u}$ ). Since the momentum $\mathbf{p}$ of a photon is proportional to the

refractive index of the medium through which it propagates, $\mathbf{p}_{i}=n_{i} \frac{h \nu}{c} \mathbf{u}$, it varies when the photon is refracted at the interface between the two media. From the second Newton's law, 
this variation of momentum is induced by a force exerted on the photon by the interface. Considering first the configuration of a downward beam incident from the upper, more refringent medium 2 to the lower, less refringent medium 1 (cf. Fig. 1a), the decrease of the photon momentum $\mathbf{p}_{1}-\mathbf{p}_{2}=\left(n_{1}-n_{2}\right) \frac{h \nu}{c} \mathbf{u}$ when crossing the interface is due to an upward force. From the third Newton's law, the force exerted by the photon on the interface, is its opposite, i.e. directed downward from the more to the less refringent medium. Considering now the reversed situation of an upward beam (cf. Fig. 1b), it is straightforward to show that the force exerted by the photon on the interface is the same, as it gains momentum when crossing the interface. This simplified corpuscular point of view shows that the direction of the force exerted on the interface by a refracted photon is independent of the direction of propagation of the electromagnetic wave.

To determine the actual radiation force exerted by the photons incident on the interface with an angle of incidence $\theta_{i}$, which is equal to the opposite of the variation per unit time of their momentum, one has to take into account the proportion of refracted and reflected photons at the interface, given by the classical reflection and transmission coefficients in electromagnetic energy $R$ and $T=1-R$ [32]. Since $n_{1} \simeq n_{2}$ in our two-fluid sample, $R$ and $T$ do not quantitatively depend on the polarization state of the incident wave [33]. Considering here the case of a downward beam incident from medium 2 to medium 1 with an angle of incidence $\theta_{i}$, as indicated in Fig. 2, it is shown in Appendix A that the radiation force $\mathbf{f}$ acting on a portion of interface of unit area is:

$$
\mathbf{f}=n_{2} \cos ^{2} \theta_{i}\left(1+R-\frac{\tan \theta_{i}}{\tan \theta_{t}} T\right) \frac{I}{c} \mathbf{n}_{2 \rightarrow 1},
$$

where $I$ is the laser beam intensity, $\theta_{t}$ the angle of refraction $\left(n_{2} \sin \theta_{i}=n_{1} \sin \theta_{t}\right)$, and $\mathbf{n}_{2 \rightarrow 1}$ is the unit vector normal to the interface and directed from medium 2 to medium 1 . Since this force is always normal to the interface whatever the angle of incidence, its intensity is called the radiation pressure (hereafter noted $\Pi$ ). At normal incidence, $\mathbf{f}$ simplifies to:

$$
\mathbf{f}\left(\theta_{i}=0\right)=2 n_{2}\left(\frac{n_{1}-n_{2}}{n_{1}+n_{2}}\right) \frac{I}{c} \mathbf{n}_{2 \rightarrow 1} .
$$

Considering now the case of an upward beam incident from medium 1 to medium 2, we obtain the expression of the radiation force per unit area just by inverting indices 1 and 2 in Eq. (1). Applying this transformation to the expression of $\mathbf{f}$ at normal incidence, Eq. (2) evidences the fact that the direction of the radiation force is independent of the beam 
direction ("up-down" invariance). It has to be noted that this property of the radiation pressure remains valid for any value of the angle of incidence.

Eq. (2) also allows us to calculate an order of magnitude of the radiation pressure. For typical conditions, i.e. excitation by a continuous wave laser beam of power $P=1 W$ focused on a $10 \mu \mathrm{m}^{2}$ surface at an interface of index contrast 0.1 , one finds $10 \mathrm{~Pa}$. This intrinsically small value shows that we need to use very soft fluid interfaces in order to be able to noticeably deform them. It also illustrates why the deformation of classical interfaces (liquid free surface, for instance) is so small $[9,16]$.

\section{B. The fluid interface used}

In order to achieve a very soft liquid interface, we consider the near-critical two-phase equilibrium state of a micellar phase of a microemulsion. The microemulsion is composed of water, oil (toluene), surfactant (sodium-dodecyl-sulfate, SDS), and co-surfactant (n-butanol1). For low concentrations of both water and surfactant, such a mixture organizes at thermodynamic equilibrium as a suspension of surfactant-coated water nanodroplets, the micelles, dispersed in an oil continuum. The used micellar phase behaves as a binary mixture of micelles and oil. It exists a critical line of consolute points with associated reverted coexistence curves [34]. For the chosen composition (mass fractions: water, 9\%, toluene, 79\%, SDS, $4 \%$, butanol, $17 \%$ ), the micelle radius is $\xi_{0}=40 \AA[35]$. This value is small enough to let the mixture be transparent in the visible window. This micellar phase of microemulsion belongs to the $d=3, n=1$ universality class of the Ising model [36]. Its critical temperature is $T_{c} \simeq 35^{\circ} \mathrm{C}$. Above $T_{c}$, this mixture separates in two micellar phases, labelled 1 and 2 , characterized by different concentrations of micelles $\phi_{1}$ and $\phi_{2}$, as indicated by the phase diagram schematically shown in Fig. 3a. In the vicinity of its consolute point, some properties of this critical two-phase mixture present scaling-law behaviors that are characteristic of the critical phenomena associated to second order phase transition of order parameter $\phi_{1}-\phi_{2}$. These quantities are the volume fraction contrast:

$$
\phi_{1}-\phi_{2}=\Delta \phi_{0}\left(\frac{T-T_{c}}{T_{c}}\right)^{\beta},
$$

with $\Delta \phi_{0}=1.458$ and $\beta=0.325$, the density contrast:

$$
\rho_{1}-\rho_{2}=\Delta \rho_{0}\left(\frac{T-T_{c}}{T_{c}}\right)^{\beta},
$$


with $\Delta \rho_{0}=284 \mathrm{~kg} \cdot \mathrm{m}^{-3}$, the refractive index contrast:

$$
n_{1}-n_{2}=\Delta n_{0}\left(\frac{T-T_{c}}{T_{c}}\right)^{\beta}
$$

with $\Delta n_{0} \simeq\left(\frac{\partial n}{\partial \rho}\right)_{T} \Delta \rho_{0}$ and $\left(\frac{\partial n}{\partial \rho}\right)_{T}=-1.22 \times 10^{-4}$, the surface tension:

$$
\sigma=\sigma_{0}\left(\frac{T-T_{c}}{T_{c}}\right)^{2 \nu}
$$

with $\nu=0.63$ and $\sigma_{0}=10^{-4} J \cdot m^{-2}$, and the capillary length:

$$
\ell_{c}=\sqrt{\frac{\sigma}{\left(\rho_{1}-\rho_{2}\right) g}}=\ell_{c 0}\left(\frac{T-T_{c}}{T_{c}}\right)^{\nu-\beta / 2},
$$

with $\ell_{c 0}=\sqrt{\frac{\sigma_{0}}{\Delta \rho_{0} g}}=190 \mu \mathrm{m}, \mathrm{g}$ being the acceleration of gravity. Our motivations to use such a medium are clearly illustrated by the following reasons. At $T-T_{c} \sim 1 K$, we find $\sigma \sim 10^{-7} \mathrm{~J}_{\mathrm{m}}^{-2}$, an extremely low value compared to that of the water free surface, which makes the interface very soft and deformable. Another obvious advantage of such a fluid interface is the possibility to tune continuously the above presented properties just by changing the sample temperature $T$.

\section{Experimental setup}

\section{Laser beam tuning}

Since the laser beam intensity depends on both the beam power and waist, the experimental setup, schematized in Fig. 4, is conceived so as to focus a vertical laser beam on the horizontal fluid interface in the sample cell $C$ and to tune the beam waist $\omega_{0}$. The beam is provided by a continuous wave $A r^{+}$laser (wavelength in vacuum $\lambda_{0}=514 n m$ ) in the $T E M_{00}$ mode. The lens $L_{1}$ forms a first intermediate waist. Selection of the upward or downward direction of the laser beam is achieved by turning the polarization of the laser light with a half-wave plate $\lambda / 2$ and directing it using the beam splitter $B S$. Beam waist tuning in $C$ is performed by moving the prism $\operatorname{Pr}$ to vary the optical path between $L_{1}$ and the long working distance microscope objectives $(\times 10)$, either $O_{1}$ (upward beam) or $O_{2}$ (downward beam). Accessible range of beam waists are $\omega_{0}=4.8-32.1 \mu m\left(\right.$ resp. $\left.\omega_{0}=3.47-11.3 \mu m\right)$ for upward (resp. downward) beam. The associated confocal parameter $z=\frac{n \pi \omega_{0}^{2}}{\lambda_{0}}$ is always 
larger than $100 \mu m$. Consequently, close to the interface, the intensity distribution $I(r, z)$ associated to the incident beam can be assumed as cylindrical along the beam axis $(O z)$ and

writes: $I(r, z) \simeq I(r)=\frac{2 P}{\pi \omega_{0}^{2}} \exp \left(-\frac{2 r^{2}}{\omega_{0}^{2}}\right)$. As the beam waist altitude varies with the optical path between $L_{1}$ and either $O_{1}$ or $O_{2}, C$ is mounted on translation stages to precisely locate the beam waist on the interface.

\section{Sample observation}

The two-phase micellar microemulsion sample is contained in a hermetical transparent glass cell, whose temperature is regulated with a stability of $\pm 0.05 K$. The phase distribution within the sample cell is shown schematically in Fig. 3 and is illustrated by the picture in Fig. 4. Interface deformations are illuminated by a white light source and are observed using a CCD video camera. The beam path through the sample can be either observed, since some fraction of the beam intensity is scattered by the micelles, or completely hidden by placing a colored glass filter on front of the camera.

\section{LINEAR REGIME OF STATIC DEFORMATION}

In order to understand why a liquid interface can be destabilized by the optical radiation pressure at a well defined beam power onset, it is necessary to get a clear view on the physics underlying the weak amplitude interface deformation obtained at much lower beam powers. This section is dedicated to this purpose.

Under the effect of the radiation pressure associated to the focused laser beam, and after a short transient, the liquid interface presents a steady, axisymmetric bell-shaped deformation. Two examples are given on top of Fig. 5a and 5b respectively for a downward and an upward propagating beam. The shape of the deformation results from the equilibrium between radiation pressure, hydrostatic pressure difference and Laplace pressure at the interface.

\section{A. Scaling approach}

As the typical length scale of optical excitation is the beam waist $\omega_{0}$, we define an optical Bond number $B o=\left(\frac{\omega_{0}}{\ell_{c}}\right)^{2}$. Bo can be experimentally varied over a wide range, from 0.01 
to 5 , by independently changing the sample temperature $T$ and the beam waist $\omega_{0}$.

On the one hand, regime interfacial effects overcome buoyancy effects in the $B o \ll 1$. Assuming that the hump of height $h$ has a typical curvature radius of $\omega_{0}$, we can get an order of magnitude of $h$ by equalling the radiation pressure $\frac{P\left(n_{2}-n_{1}\right)}{c \omega_{0}^{2}}$ (using Eq. $2, I \sim \frac{P}{\omega_{0}^{2}}$ and $\left.n_{1} \simeq n_{2}\right)$ and the Laplace pressure $\sigma \frac{h}{\omega_{0}^{2}}$. We find $h \sim \frac{P\left(n_{2}-n_{1}\right)}{\sigma c}$.

On the other hand, gravitational effects dominate interfacial effects in the $B o \gg 1$ regime. By equalling the hydrostatic pressure step at the interface $\left(\rho_{1}-\rho_{2}\right) g h$ and the radiation

pressure, we get $h \sim\left(\frac{\partial n}{\partial \rho}\right)_{T} \frac{P}{\omega_{0}^{2}} \frac{1}{g c}$. Note that in the $B o \ll 1$ regime $h$ scales as $P$, whereas $h$ scales as $I$ in the $B o \gg 1$ regime.

\section{B. Linear model}

The axisymmetric interface deformations are naturally described in cylindrical coordinates $(r, \varphi, z)$ centered along the beam axis $(O z)$ by the height $h(r)$ of the deformed interface shifted from its flat position at rest, or equivalently, by the angle $\theta_{i}(r)=\arctan \left(h^{\prime}(r)\right)$ between the deformed interface and the horizontal (see Fig. 2). Assuming that the equilibrium shape of the interface is only determined by the balance between the electromagnetic radiation pressure, the hydrostatic pressure difference and the Laplace pressure at the interface, i.e. neglecting electrostriction [10] and thermo-capillary effects [21], the steady interface profile should be accurately described by the following equation:

$$
\left(\rho_{1}-\rho_{2}\right) g h(r)-\sigma \kappa(r)=\Pi\left(r, z, \theta_{i}\right)
$$

where $\kappa(r)=\frac{1}{r} \frac{d}{d r}\left(r \sin \theta_{i}(r)\right)=\frac{1}{r} \frac{d}{d r}\left(\frac{r h^{\prime}(r)}{\sqrt{1+h^{\prime 2}(r)}}\right)$ is the curvature of the interface and $\Pi\left(r, z, \theta_{i}\right)$ the radiation pressure given by Eq. (1).

Small amplitude deformations are defined as those for which $\left|h^{\prime}(r)\right|=\left|\tan \theta_{i}(r)\right| \simeq$ $\left|\theta_{i}(r)\right| \ll 1$. This condition has three consequences. (i) $\kappa(r) \simeq \frac{1}{r} \frac{d}{d r}\left(r h^{\prime}(r)\right)$. (ii) The laser beam can be considered as impinging the interface at normal incidence. (iii) Although the beam propagation is modified by the lens effect of the deformed interface [37], these perturbations do not modify the interface shape in turn (no propagation-deformation feedback). This means that the radiation pressure is only due to the incident, parallel laser beam: $\Pi=\Pi\left(r, \theta_{i}=0\right)$. Thus, steady deformations of small amplitude should be described by the 
linearized expression of Eq. (8). This linear approximation leads to:

$$
\begin{aligned}
& \left(\rho_{1}-\rho_{2}\right) g h(r)-\sigma \frac{1}{r} \frac{d}{d r}\left(r h^{\prime}(r)\right) \\
& =-\frac{n_{i}}{c} \frac{n_{2}-n_{1}}{n_{1}+n_{2}} \frac{4 P}{\pi \omega_{0}^{2}} \exp \left(-\frac{2 r^{2}}{\omega_{0}^{2}}\right),
\end{aligned}
$$

where $i=1$ (resp. $i=2$ ) corresponds to an upward (resp. downward) beam crossing the interface from medium 1 to medium 2 (resp. from medium 2 to medium 1 ).

In the $B o \gg 1$ regime, the solution of Eq. (9) is simply:

$$
h(r)_{B o \gg 1}=-\left(\frac{\partial n}{\partial \rho}\right)_{T} \frac{n_{i}}{n_{1}+n_{2}} \frac{4 P}{\pi g c \omega_{0}^{2}} \exp \left(-\frac{2 r^{2}}{\omega_{0}^{2}}\right) .
$$

Since $n_{1} \simeq n_{2}$ near criticality, the height of the hump is

$$
h(r=0)_{B o \gg 1} \simeq\left(\frac{\partial n}{\partial \rho}\right)_{T} \frac{2 P}{\pi g c \omega_{0}^{2}},
$$

as predicted by the scaling approach. Moreover, Eq. (10) shows that the curvature radius of the hump scale as $\omega_{0}$, as assumed in the scaling approach.

In the $B o \ll 1$ regime, the solution of Eq. (9) reads:

$$
\begin{aligned}
& h(r)_{B o \ll 1}=-\frac{n_{i}}{c} \frac{n_{2}-n_{1}}{n_{2}+n_{1}} \frac{P}{2 \pi \sigma} \\
& \quad\left[E_{1}\left(\frac{8}{\Gamma B o}\right)-E_{1}\left(\frac{2 r^{2}}{\omega_{0}^{2}}\right)-\ln \left(\frac{\Gamma B o}{4} \frac{r^{2}}{\omega_{0}^{2}}\right)\right],
\end{aligned}
$$

where $E_{1}$ is the 1-argument exponential function $\left(E_{1}(x)=\int_{x}^{+\infty} \frac{e^{-u}}{u} d u\right)$ and $\Gamma=1.781$ is the Euler constant. Indeed, by integrating Eq. (9) we find:

$$
\begin{aligned}
& h(r)_{B o \ll 1}=-\frac{n_{i}}{c} \frac{n_{2}-n_{1}}{n_{2}+n_{1}} \frac{P}{2 \pi \sigma} \\
& {\left[E_{1}\left(\frac{\omega_{b c}^{2}}{\omega_{0}^{2}}\right)-E_{1}\left(\frac{2 r^{2}}{\omega_{0}^{2}}\right)-\ln \left(\frac{2 r^{2}}{\omega_{0}^{2}}\right)\right],}
\end{aligned}
$$

where $\omega_{b c}$ is a radius, large compared to $\omega_{0}$, which is defined by the boundary condition $h\left(r=\omega_{b c}\right)_{B o \ll 1}=0$. For integration we also assumed $\frac{\partial h_{B o \ll 1}}{\partial r}(r=0)=0$. To determine $\omega_{b c}$, we solve Eq. (9) using the Fourier-Bessel transform $h(r)=\int_{0}^{\infty} \widetilde{h}(k) J_{0}(k) k d k$, where $J_{0}$ is the $0^{t h}$-order Bessel $J$ function. The general expression of $h(r)$ becomes:

$$
h(r)=-\frac{n_{i}}{c} \frac{n_{2}-n_{1}}{n_{2}+n_{1}} \frac{P}{\pi} \int_{0}^{\infty} \frac{\exp \left(-\frac{k^{2} \omega_{0}^{2}}{8}\right)}{\left(\rho_{1}-\rho_{2}\right) g+\sigma k^{2}} J_{0}(k r) k d k .
$$


The nice aspect of Eq. (14) is that we can obtain an analytical expression of $h(r=0)$. we find:

$$
h(r=0)=h(r=0)_{B o \gg 1} \times F(B o),
$$

where $F(B o)$ is given by:

$$
F(B o)=\frac{B o}{8} \exp \left(\frac{B o}{8}\right) E_{1}\left(\frac{B o}{8}\right)
$$

As $E_{1}(x \ll 1) \simeq-\ln (\Gamma x)+x$, we find for $B o \ll 1$ :

$$
h(r=0)_{B o \ll 1}=-\frac{n_{i}}{c} \frac{n_{2}-n_{1}}{n_{2}+n_{1}} \frac{P}{2 \pi \sigma} \ln \left(\frac{8}{\Gamma B o}\right),
$$

which leads to $\omega_{b c}=2 \sqrt{2} \ell_{c} / \Gamma$, and thus to the expression of $h(r)$ given by Eq. (12). This exact calculation reveals the $B o$ correction to the $B o \ll 1$ scaling law for $h$ obtained by the

scaling approach. On the other hand, as $E_{1}(x)_{x \rightarrow \infty} \rightarrow 0$ and $\frac{\omega_{b c}^{2}}{\omega_{0}^{2}}=\frac{8}{\Gamma B o} \gg 1$, the solution of Eq. (12) close to the beam axis writes:

$$
h\left(r \ll \omega_{0}\right)_{B o \ll 1} \simeq-\frac{n_{i}}{c} \frac{n_{2}-n_{1}}{n_{1}+n_{2}} \frac{P}{2 \pi \sigma}\left[\ln \left(\frac{8}{\Gamma B o}\right)-\frac{2 r^{2}}{\omega_{0}^{2}}\right] .
$$

Consequently, the curvature radius of the hump scales as $\omega_{0}$.

\section{Comparison with experiments}

We analyzed the hump height $h(r=0)$ of small amplitude deformations obtained using an upward propagating beam, for several beam waists and power values, and we compared our measurements to the above presented theoretical predictions.

In the $B o \ll 1$ regime the variations of $h(r=0)$ versus $\frac{P\left(n_{2}-n_{1}\right)}{\sigma c}$, plotted in Fig. 6, are found to be linear and independent of $\omega_{0}$. This behavior confirms the validity of Eq. (17) for the $B o \ll 1$ regime. Moreover, in the bottom of Fig. 5b, the digitized shapes of interface deformations corresponding to the above snapshots, are compared to the predicted shapes (solid lines) computed using Eq. (12). The observed satisfactory agreement shows that we retrieved the expected behavior for the $B o \ll 1$ regime [18].

To illustrate the $B o \gg 1$ regime, the variations of $h(r=0)$ are plotted versus $\frac{\left(n_{2}-n_{1}\right)}{\left(\rho_{2}-\rho_{1}\right)} \frac{P}{g c \omega_{0}^{2}}$ in Fig. 7 for several values of $B o$ that are larger than 2.0. A linear behavior with a slope independent of temperature is observed. This confirms the validity of Eq. (11) predicted for $B o \gg 1$. 


\section{D. "Up-down" invariance}

According to Eq. (2), the interface deformations at normal incidence, i.e. their direction, shape and height, should not depend on the direction of the beam because $n_{1} \simeq n_{2}$ (see also Fig. 5). Given any value of Bo, Eqs. (15-16) show that the hump height $h(r=0)$ can be expressed in a synthetic fashion as a function of both $h(r=0)_{B o>>1}$ and $B o$. To evidence this "up-down" invariance, we plotted in Fig. 8 the variations of the normalized hump

height $\tilde{h}(B o)=\frac{h(r=0)}{\left.h(r=0)\right|_{B o \gg 1}}$ versus $B o$, measured for both upward and downward directions of propagation of the laser beam and for all the experimentally investigated values of $B o$. Both data sets collapse onto the solid line representing the behavior of $F(B o)$ predicted by Eq. (16). This agreement confirms the theoretically predicted invariance of the deformation with respect to the beam direction at small deformation amplitude.

\section{E. Up-down invariance breakdown}

We end this section dedicated to the linear regime of deformation by characterizing the limits of this regime, and more precisely the up-down invariance breakdown in the $B o \ll 1$ regime. Indeed, at moderate beam power, the interface shape starts to noticeably depend on the direction of propagation of the laser beam.

Let us first consider a laser beam propagating upward. In the $B o \ll 1$ regime, and for values of the beam power of the order of $6 \frac{\sigma c \omega_{0}}{n_{2}-n_{1}}$, the interface exhibits a tether-like shape, as shown by the right-hand picture of Fig. 9, whereas the height $h(r=0)$ deviates from the linear relationship $h(r=0) \propto \frac{P\left(n_{2}-n_{1}\right)}{\sigma c}$ illustrated by the straight line. Such shapes, which may be attributed either to the coupling between the deformation of the interface and the resulting refraction of the laser beam, or to optical nonlinearity induced by electro-osmosis, shall deserve further investigation.

The situation is totally different for a laser propagating downward. By increasing progressively the beam power $P, h(r=0)$ increases first linearly with $P$, as described in Sec. III C. Then, $h(r=0)$ presents a sharp increase as $P$ approaches a well defined threshold value called $P_{\uparrow}$, but remains finite and stationnary, as shown in Fig. 9. $P_{\uparrow}$ is defined as the threshold value of the beam power above which the interface is no longer stable. Instability leads to the formation of a very long cylindrical jet, as shown in the left-hand picture of 
Fig. 9. Its diameter is of the order of $\omega_{0}$ whereas its length is typically a few hundreds of micrometers. This instability threshold as well as its possible origin are the purpose of the next two sections. The physics of the jet will be the subject of a future article.

\section{INTERFACE INSTABILITY}

We studied this interface instability mainly in the $B o \ll 1$ regime, where surface effects overcome gravity effects.

\section{A. Beam power behavior just below the instability threshold}

We measured the dependance of $P_{\uparrow}$ with respect to the beam waist $\omega_{0}$ and temperature difference $T-T_{c}$ in the range $\omega_{0}=3.5-11 \mu \mathrm{m}$ and $T-T_{c}=1.5-15 \mathrm{~K}$, corresponding to $B o=0.006-0.54$. $P_{\uparrow}$ was determined with an accuracy of $\pm 10 \mathrm{~mW}$. The corresponding variations of $P_{\uparrow}$ versus $\omega_{0}$ are plotted in Fig. 10a. For each value of $T-T_{c}, P_{\uparrow}$ is found to vary linearly with $\omega_{0}$. The variation of $P_{\uparrow}$ versus $T-T_{c}$ for $\omega_{0}=3.5 \mu m$ are plotted in Fig. $10 \mathrm{~b}$ in $\log$-log scales. It is found to be compatible with a power law behavior $\left(T-T_{c}\right)^{1.01 \pm 0.05}$.

\section{B. Dimensional analysis and empirical scaling law}

In the $B o \ll 1$ regime, dimensional analysis shows that $\frac{P_{\uparrow}}{\sigma c \omega_{0}}$ is a function of $\left(n_{2}-n_{1}\right)$ only. Given the power law behavior in $\left(T-T_{c}\right)$ of $P_{\uparrow}$ shown in Fig. 10b, and considering Eq. (6) and Eq. (4), we can confidently assume this function to behave as a power law of $n_{2}-n_{1}: P_{\uparrow} \propto \sigma c \omega_{0}\left(n_{2}-n_{1}\right)^{x}$, and find $x=(-0.77 \pm 0.13) \simeq-1$. The validity of this empirical scaling law is confirmed by the variations of the quantity $\frac{P_{\uparrow}\left(n_{2}-n_{1}\right)}{\sigma c}$ versus $\omega_{0}$ shown in Fig. 11. The best linear fit of this master curve gives:

$$
P_{\uparrow}=\beta \frac{\sigma c \omega_{0}}{n_{2}-n_{1}} \text { with } \beta=6.3 \pm 0.3
$$

\section{A possible opto-hydrodynamic mechanism for the interface instability}

As recently proposed in [30], a possible cause for the up-down invariance breakdown in the regime of large deformation could be the fact that light rays propagating downward in 
the phase of largest refractive index can encounter total reflection when the laser impinges a highly deformed interface; note that no such behavior occurs when laser propagates upward in the phase of smallest optical index. Increasing progressively $P$, total reflection would occur first along the circle formed by the inflexion points of the interface shape, where $\theta_{i}$ is maximum. This initially small amount of reflected light would be refocused towards the beam axis, resulting in an increase of the radiation pressure at the tip of the deformation. This pressure variation would in turn stretch the interface deformation, and consequently increase the area of the part of the interface which totally reflects light. This positive feedback could thus be a realistic mechanism for this opto-hydrodynamic instability. As illustrated in Fig. 12, the plausibility of this mechanism is supported by the observation of light focusing within the deformation during the instability growth and the following jet formation.

This mechanism is based on the assumption that the maximum of $\theta_{i}, \theta_{i \text { max }}$, equals the angle of total reflection $\theta_{T R}=\arcsin \frac{n_{1}}{n_{2}}$ at the instability onset. We test the validity of this hypothesis by measuring precisely the shape of the interface just below the instability threshold in order to determine the maximum of the incidence angle along the interface. Note that, given Eq. (5), one has

$$
\theta_{T R}=\arcsin \frac{n_{0}-\frac{\Delta n_{0}}{2}\left(\frac{T-T_{c}}{T_{c}}\right)^{0.325}}{n_{0}+\frac{\Delta n_{0}}{2}\left(\frac{T-T_{c}}{T_{c}}\right)^{0.325}},
$$

where $n_{0}=1.464$. $\theta_{T R}$ is expected to vary between $85^{\circ}$ and $82^{\circ}$ in the investigated temperature range $T-T_{c}=2-10 K$. These very large values arise from the smallness of the index contrast in our near-critical two-phase system.

\section{Maximum angle of incidence just below the instability threshold}

\section{Interface shape digitization}

We captured and digitalized the interface profile just below the instability threshold $\left(\left|P-P_{\uparrow}\right| \leq 10 \mathrm{~mW}\right)$ in the range of beam waists $\omega_{0}=3.47-6.95 \mu \mathrm{m}$ and for temperatures $T-T_{c}=2-10 K$. In terms of $B o$ number, the investigated range is $B o=0.008-0.14$. Since we were interested in the slope of the interface shape, particularly around its inflexion points along its sides, and since the determination of a slope is very sensitive to digitization 
noise, we employed a special process for digitizing the interface shape. We computed the radial gradient of the raw picture (i.e. along the $r$ axis), and then we detected the intensity maximum along each pixel line. Thus, the left and right sides of the interface deformation are defined in an unusual manner as $r_{l}(h)$ and $r_{r}(h)$, as illustrated in Fig. 13. For precision purpose, the tip of the interface deformation is more classically obtained by computing the vertical gradient of the raw picture (i.e. along the $z$ axis) and detecting the intensity maximum of each pixel column. It is thus defined as $h(r)$.

\section{Determination of the angle of incidence around the inflexion points of the interface shape}

In order to precisely determine the slope of the digitized interface shape around its inflexion point, we fitted each of its sides $r_{l}(h)$ and $r_{r}(h)$ using two different polynomial functions of third degree $Q_{l}(h)$ and $Q_{r}(h)$, which exhibit a single inflexion point. The angle $\theta_{i}(r)$ between the interface and the horizontal is obtained on the left and the right side of the deformation using respectively $\theta_{i}(r)\left(^{\circ}\right)=-90-\arctan \left(\frac{d Q_{l}}{d h}(h(r))\right)$ and $\theta_{i}(r)\left(^{\circ}\right)=90-\arctan \left(\frac{d Q_{r}}{d h}(h(r))\right)$ (see Fig. 13). Although we could not estimate precisely the accuracy of this method for the determination of the angle of incidence, we checked that fits using polynomial functions of higher degrees gave close values for the maximum of the angle of incidence, and we concluded that its accuracy was of the order of $\pm 2^{\circ}$.

\section{Maximum angle of incidence just below the instability threshold}

Calling $\theta_{i l \max }<0$ (resp. $\theta_{i \mathrm{r} \max }>0$ ) the minimum (resp. maximum) of the angle of incidence along the left (resp. right) side of the interface shape, we define the maximum angle of incidence as $\theta_{i \max }=\frac{\theta_{i \mathrm{r} \max }-\theta_{i 1 \max }}{2}$. The variations of $\theta_{i \max }$ measured just below the instability threshold are plotted in Fig. 14 versus $T-T_{c}$ for several values of $\omega_{0}$, together with the variation with $T-T_{c}$ of the angle $\theta_{T R}$ predicted for total reflection. While $\theta_{i \max }$ is found to fluctuate around $72^{\circ}$ with no noticeable dependance with respect to $T-T_{c}$, it is significantly smaller than $\theta_{R T}$ by $10-15^{\circ}$.

In Fig. 15, the variations of the same measurements of $\theta_{i \max }$ are plotted as a function of $\omega_{0}$ for several values of $T-T_{c}$. $\theta_{i}$ max is also found to exhibit no noticeable dependance with respect to $\omega_{0}$. 


\section{E. Discussion}

The $10-15^{\circ}$ gap between $\theta_{i \max }$ and $\theta_{R T}$ has a dramatic incidence on the validity of the proposed mechanism which assumes that instability is triggered by total reflection of light within the deformed interface. As a matter of fact, since $n_{1} \simeq n_{2}$, the Fresnel reflection coefficient of energy $R$ is very small at any angle of incidence, except very close to $\theta_{T R}$, whatever the polarization of the incident wave [33]. This is illustrated in Fig. 16a for the particular case of a transverse electric (TE) polarized wave. In particular, $R$ is of the order of $10^{-3}$ for $\theta_{i} \simeq 72^{\circ}$. As a consequence, instability occurs whereas only a very small amount of the incident energy is reflected towards the tip of the deformation. Although this instability mechanism has not to be definitely rejected, the measurements of $\theta_{i \max }$ presented in Figs. 14-15 indicate that the scenario leading to the interface instability is more complex.

To determine more precisely the actual influence of the reflected light on the interface shape at the instability threshold, we computed the additional contribution of the light partially reflected at the interface to the radiation pressure within the frame of geometrical optics. This computation, presented in Appendix B, shows that additional radiation pressure contribution of the partially reflected light is indeed small compared to the radiation pressure of the incident light everywhere along the interface except very close to the beam axis, where focusing of the reflected light is probably overestimated. Consequently, the interface shape should be accurately described by considering the radiation pressure of the incident light only.

One way for retaining the idea of reflection induced instability is inspired by RayleighBénard convection in pure fluids near the liquid-vapor critical point [38]. In such supercritical fluids, the onset of convection can be reached without increasing neither the temperature difference between the top and the bottom of the fluid layer, nor its height, but by increasing only the thermo-mechanical susceptibility of the fluid, i.e. its coefficient of thermal expansion at constant pressure $\alpha_{P}$, by reducing $\langle T\rangle-T_{c}\left(\langle T\rangle\right.$ is the average sample temperature, $T_{c}$ is the critical temperature). In the present experiment, the susceptibility to be considered would be the rate of variation of the Fresnel reflection coefficient in energy $R$ with respect to $\theta_{i}, \frac{1}{R} \frac{d R}{d \theta_{i}}$, whose behavior is plotted in Fig. 16b. This quantity, which can be considered as the relative variation of the reflected energy when $\theta_{i}$ fluctuates around is steady-state value, corresponds to the susceptibility of the interface shape to angle fluctuations. As illustrated 
in Fig. 16b, it increases strongly when $\theta_{i}$ approaches $\theta_{T R}$, and takes values comparable to unity when $\theta_{i}$ approaches $72^{\circ}$. At such values of $\theta_{i}$, small fluctuations of the angle of incidence could be amplified due to the shape changes of the interface induced by the large fluctuations of reflected energy towards the tip of the deformation.

Note finally that $\frac{1}{R} \frac{d R}{d \theta_{i}}$ is actually independent of temperature (except close to $\theta_{R T}$ ), in particular around $\theta_{i}=72^{\circ}$. This can be related to the fact that $\theta_{i \text { max }}$ at instability threshold does not depend on temperature (see Fig. 14).

\section{F. Summary}

We proposed that interface instability could be triggered by a radiation pressure increase due to the total reflection of light towards the tip. We showed that at the instability onset, only a small amount of the incident electromagnetic energy is reflected towards the tip, because the maximum angle of incidence along the deformed interface is smaller than the angle of total reflection, in apparent contradiction with the proposed model. Consequently, if the instability mechanism still involves the light reflected towards the tip, it is more complex than previously expected. Nevertheless, assuming that the instability mechanism should be intimately linked to the interface shape close to the instability threshold, we need to know whether such a simple model as Eq. (8) describes well the interface shape close to the instability threshold and whether it can predict the onset of instability. This is the purpose of the next part.

\section{STATIONARY INTERFACE DEFORMATIONS BELOW THE INSTABILITY THRESHOLD}

\section{A. Simple model valid in the $B o \ll 1$ regime}

Let us assume deformations of arbitrary amplitude caused by a downward propagating beam. Then, considering Eq. (1), Eq. (8) simplifies in the $B o \ll 1$ regime to

$$
\begin{aligned}
& \sigma \frac{1}{r} \frac{d}{d r}\left(r \sin \theta_{i}(r)\right)=-n_{2} \cos ^{2} \theta_{i} \\
& \quad\left(1+R-\frac{\tan \theta_{i}}{\tan \theta_{t}} T\right) \frac{2 P}{\pi \omega_{0}^{2} c} \exp \left(-\frac{2 r^{2}}{\omega_{0}^{2}}\right),
\end{aligned}
$$


for incidence angles $\theta_{i}$ smaller than the angle of total reflection $\theta_{R T}=\arcsin \left(\frac{n_{2}}{n_{1}}\right)$. Following [39], we use the dimensionless coordinates $\tilde{r}=\frac{\sqrt{2} r}{\omega_{0}}$ and $\tilde{h}=\frac{\sqrt{2} h}{\omega_{0}}$. Since $n_{2} \cos ^{2} \theta_{i}\left(1+R-\frac{\tan \theta_{i}}{\tan \theta_{t}} T\right) \simeq 4\left(n_{2}-n_{1}\right)\left(\frac{\cos \theta_{i}}{\cos \theta_{i}+\cos \theta_{t}}\right)^{2}$ for $n_{2} \simeq n_{1}$, we rewrite Eq. (21) as:

$$
\frac{d \sin \theta_{i}}{d \tilde{r}}=-\frac{\sin \theta_{i}}{\tilde{r}}-K\left(\frac{\cos \theta_{i}}{\cos \theta_{i}+\cos \theta_{t}}\right)^{2} e^{-\tilde{r}^{2}}
$$

where $K=\frac{4 \sqrt{2}\left(n_{2}-n_{1}\right) P}{\pi \sigma c \omega_{0}}$. This first order non linear differential equation is solved numerically with $\theta_{i}(r=0)=0$ as initial condition. The corresponding dimensionless interface profile $\tilde{h}(\tilde{r})$ is obtained by numerical integration of:

$$
\frac{d \tilde{h}}{d \tilde{r}}=\frac{\sin \theta_{i}}{\sqrt{1-\sin ^{2} \theta_{i}}}
$$

with $\tilde{h}(r=0)=0$ as initial condition. As gravity effects are not taken into account here, Eq. (23) cannot predict accurately the absolute height of the interface deformation, but only its shape near the beam axis, where curvature effects overcome gravitational effects.

\section{B. Confrontation to experiments just below the instability threshold}

\section{Comparison between expected and measured interface shapes}

To reproduce numerically the measured steady interface profiles just below the instability threshold using Eq. (22), we did not use the experimental values of $P_{\uparrow}$, since they are slightly noisy (see Fig. 11). We rather choose to fix $P$ so that the computed value of $\theta_{i \text { max }}$ is equal to $72^{\circ}$, which corresponds to the average value of the maximum angle of incidence $\theta_{i \max }$ measured just below the instability threshold (see Figs. 14-15).

The comparison between computed and experimental interface profiles obtained at $T-$ $T_{c}=8 \mathrm{~K}$ is presented in Fig. 17. This confrontation is made in reduced coordinates and for several values of beam waists. Experimental interface shapes are found to be systematically wider than the predicted ones. However, satisfactory coincidence is met at the largest values of $\omega_{0}$, whereas disagreement is more pronounced at the smallest values of $\omega_{0}$. This trend is found for each investigated temperature. We now discuss three possible causes for this mismatch between predicted and experimental deformation shapes.

Gravity effects should appear at large values of $B o$, i.e. for large values of $\omega_{0}$, and thus are to be rejected. 
Thermocapillary effects, which are expected to scale as $P$ [21], i.e. as $P_{\uparrow}$ at instability threshold (Eq. 19), should decrease with decreasing $\omega_{0}$, and thus are to be rejected too.

Finally, the interface tip could be deformed by the additional radiation pressure contribution of the light partially reflected at the interface. A dimensional analysis shows that, in the $B o \ll 1$ regime, the resulting perturbation of the angle of incidence should be a function of $P / P_{\uparrow}$ and $\left(n_{2}-n_{1}\right)$ only, i.e. of $\left(n_{2}-n_{1}\right)$ only at instability threshold $\left(P=P_{\uparrow}\right)$. However, the interface shape mismatch between experiment and numerical simulation was not found to depend on temperature. Moreover, it is shown in Appendix B that the amount of reflected energy at the instabilty threshold is very small and results in a very weak additional contribution to the radiation pressure. Consequently, this mismatch remains presently unexplained.

This nonlinear model of steady interface deformation can thus be confidently used to simulate the interface shapes at the instability threshold for large values of $\omega_{0}$ with the aim of determining the instability mechanism.

Having confronted the experimental and computed interface shapes just below the instability threshold, we now compare the experimental and computed beam power thresholds $P_{\uparrow}$ required to trigger the instability.

2. Comparison between experimental and computed threshold values of the beam power just below the instability threshold

Since the maximum angle of incidence just below instability threshold is smaller than the angle of total reflection, one would expect that the simple model of total internal reflection induced instability presented in Sec. IV C, which assumes that onset corresponds to $\theta_{i \max }=$ $\theta_{R T}$, would predict too large threshold values for the beam power $P_{\uparrow}$ at the onset. This is not the case.

Indeed, we first note that Eq. (22) is numerically stable for $\theta_{i \max }>72^{\circ}$, in fact up to $\theta_{i \max }=\theta_{T R}$, whereas interface shapes are experimentally found to be unstable for $\theta_{i \max } \gtrsim 72^{\circ}$. Consequently, Eq. (22) does not contain the mechanism of opto-hydrodynamic instability of the interface explaining an instability onset at $\theta_{i \max } \simeq 72^{\circ}$.

Let us compute the threshold value $P_{T R}$ of the beam power $P$ at which the maximum of the function $\theta_{i}(r)$, solution of Eq. (21), reaches $\theta_{T R}$. The analytic solution of a linearization 
of Eq. (21) gives $P_{T R}=6.9 \frac{\sigma c \omega_{0}}{n_{2}-n_{1}}$ [30], whereas the numerical solution of Eq. (22) gives $P_{T R}=6.5 \frac{\sigma c \omega_{0}}{n_{2}-n_{1}}$. Both theoretical prefactors of these theoretical scaling laws are very close to the experimental prefactor given in Eq. (19) (relative differences less than $10 \%$ and 3\%, respectively).

Let us now compute the value $P_{72^{\circ}}$ of the beam power $P$ at which the maximum of the function $\theta_{i}(r)$ solution of Eq. (22), $\theta_{i \max }$, reaches $72^{\circ}$. We find that the relative difference between $P_{T R}$ and $P_{72^{\circ}}$ decreases from $3.4 \%$ at $T-T_{c}=2 \mathrm{~K}$ to $2.4 \%$ at $\mathrm{T}-T_{c}=10 \mathrm{~K}$. A so small relative difference (in fact smaller than the experimental uncertainty of the $P_{\uparrow}$ measurements) explains why the simple model of total internal reflection induced instability presented in Sec. IV C gives a prediction of the value of the beam power at onset $P_{T R}$ in quantitative agreement with measurements of $P_{\uparrow}$, whereas the instability criterium assumed by this model basically overestimates $P_{\uparrow}$.

\section{CONCLUSION}

Using a soft interface between the coexisting phases of a near-critical fluid mixture, we studied the interface deformations induced by the electromagnetic radiation pressure of a focused cw laser beam. By varying the sample temperature, the power and the waist of the beam, and making use of the universal power-law behaviors of the physical properties of the mixture, we showed in Sec. III C that the shapes of the deformations result principally from the equilibrium between the radiation pressure, the hydrostatic pressure difference and the Laplace pressure at the interface. Using a simple linear model of static equilibrium of the interface (Eq. 9), we were able to explain the observed hump height variations for any value of the Bond number $B o$ in the linear regime of deformation. Furthermore, we showed that the deformations where independent of the direction of propagation of the laser. The linear regime of deformation seems to be thus well understood.

When the laser propagates from the more to the less refringent phase, and at moderate beam power, we observed an instability of the interface leading to the formation of a long jet acting as a wave-guide for the laser beam. We proposed in Sec. IV C that the total internal reflection of the incident light on the highly deformed interface could be at the origin of this instability. Using a simple nonlinear model of static equilibrium of the interface taking account of the radiation pressure of the incident light only (Eq. 22), we could explain the 
observed beam power threshold of the instability as well as the deformation tip shapes for the larger waists observed just below the instability onset. According to this model, the instability should occur when the interface slope reaches the angle of total reflection $\theta_{T R}$. We measured the maximum incidence angle along the interface $\theta_{\mathrm{i} \text { max }}$ just below the instability threshold and found that it was significantly smaller than $\theta_{T R}$. Furthermore, (Eq. 22) does not present any unstable behavior up to $\theta_{\mathrm{i} \max }=\theta_{T R}$. Thus, we are in a paradoxical situation, i.e. we can satisfactorily model the interface shape just below the instability threshold, whereas the model we use does not contain any instability mechanism. To definitely determine the relevance of this model of reflection induced instability, a numerical study of the stability of the deformed interface should be developed by including the additional radiation pressure contribution of the light partially reflected at the interface. It should couple a fully nonlinear and unsteady two-phase hydrodynamic model of the interface dynamics and the computation of the propagation of electromagnetic waves through the interface between both dielectric liquids.

\section{Acknowledgments}

The authors thank W. Zhang for fruitful discussion. They also thank M. Winckert and J. Plantard for technical assistance. This work was partly supported by Centre National de la Recherche Scientifique and Conseil Régional d'Aquitaine.

\section{APPENDIX A: SIMPLE DETERMINATION OF THE RADIATION FORCE AT ANY ANGLE OF INCIDENCE}

The aim of this appendix is to compute the radiation force acting on an interface under any angle of incidence. We consider two dielectric media, separated by an interface of arbitrary shape, with different refractive indices $n$. The indices $i$ and $t$ refer to incidence and transmission, and $\theta_{i}$ and $\theta_{t}$ are respectively the incident and transmission angles. The momentum carried by the incident light is not necessarily conserved when the beam crosses the interface separating these two dielectrics. The resulting discontinuity in momentum gives birth to the radiation pressure applied to the interface. Let us call $\mathbf{t}$ and $\mathbf{n}$ the tan-

gent and the normal directions to the interface at the location where the light impinges the 
interface (see Fig. 18). We deduce the following properties: (i) an incident photon gives the momentum $\frac{n_{i} h \nu}{c}\left(\sin \theta_{i} \mathbf{t}+\cos \theta_{i} \mathbf{n}\right)$ to the interface, (ii) a reflected photon picks the momentum $\frac{n_{i} h \nu}{c}\left(\sin \theta_{i} \mathbf{t}-\cos \theta_{i} \mathbf{n}\right)$ to the interface, (iii) a transmitted photon picks the momentum $\frac{n_{t} h \nu}{c}\left(\sin \theta_{t} \mathbf{t}+\cos \theta_{t} \mathbf{n}\right)$ to the interface. To calculate the radiation pressure associated to a light beam, we denote $N$ the number of photons impinging the interface per unit time and unit surface area, and $R\left(\theta_{i}, \theta_{t}\right)$ and $T\left(\theta_{i}, \theta_{t}\right)=1-R\left(\theta_{i}, \theta_{t}\right)$ the classical Fresnel coefficients of reflection and transmission of electromagnetic energy. Consequently, the momentum variation $d \mathbf{Q}$ of the photons incident on an interface element of area $d S$ during the time $d t$ is $d \mathbf{Q}=d \mathbf{Q}_{\|}+d \mathbf{Q}_{\perp}$, where the symbols $\|$ and $\perp$ correspond respectively to its components in the $\mathbf{t}$ and $\mathbf{n}$ directions:

$$
\begin{aligned}
d \mathbf{Q} & =d \mathbf{Q}_{\|}+d \mathbf{Q}_{\perp} \\
& =\left(n_{i} \sin \theta_{i}-\left(R n_{i} \sin \theta_{i}+T n_{t} \sin \theta_{t}\right)\right) \frac{N h \nu}{c} d S d t \mathbf{t} \\
& +\left(n_{i} \cos \theta_{i}-\left(-R n_{i} \cos \theta_{i}+T n_{t} \cos \theta_{t}\right)\right) \frac{N h \nu}{c} d S d t \mathbf{n}
\end{aligned}
$$

Accordingly, $d \mathbf{Q}_{\|}$reads:

$$
d \mathbf{Q}_{\|}=\left(n_{i} \sin \theta_{i}(1-R)-n_{t} \sin \theta_{t} T\right) \frac{N h \nu}{c} d S d t \mathbf{t}
$$

As $n_{i} \sin \theta_{i}=n_{t} \sin \theta_{t}, d \mathbf{Q}_{\|}=\mathbf{0}$. There is no momentum transfer parallel to the interface. Consequently, one has:

$$
d \mathbf{Q}=d \mathbf{Q}_{\perp}=n_{i} \cos \theta_{i}\left(1+R-\frac{\tan \theta_{i}}{\tan \theta_{t}} T\right) \frac{N h \nu}{c} d S d t
$$

Classically, the laser intensity $I$ is defined as $I=N_{0} h \nu$, where $N_{0}$ is the flux of photons though the beam section. As the incident wave is tilted by an angle $\theta_{i}$ at the interface, one gets $N=N_{0} \cos \theta_{i}$. We deduce that the radiation force $\mathbf{f}$ acting on a portion of interface of unit area is given by:

$$
\mathbf{f}=n_{i} \cos ^{2} \theta_{i}\left(1+R-\frac{\tan \theta_{i}}{\tan \theta_{t}} T\right) \frac{I}{c} \mathbf{n} .
$$

Consequently, $\mathbf{f}$ is always normal to the interface. Moreover, by considering the expressions of the reflection and transmission coefficients $R$ and $T$ [32], it can be easily shown that the optical radiation force is directed towards the dielectric medium of lowest refractive index. 


\section{APPENDIX B: COMPUTATION OF THE ADDITIONAL CONTRIBUTION TO THE RADIATION PRESSURE OF THE LIGHT PARTIALLY REFLECTED AT THE INTERFACE WITHIN THE FRAME OF RAY OPTICS}

The aim of this appendix is to compute the additional radiation pressure acting on the interface due the light partially reflected at the interface just below the instability threshold. The exact computation of the electromagnetic field along the interface resulting from diffraction and one or several partial reflections requires a heavy numerical effort. Instead, we propose to evaluate at each point $A_{1}$ along the interface, the contributions to the radiation pressure (i) of the light directly incident (called hereafter the direct ray) and (ii) of the light having previously been reflected once at another point $A_{2}$ along the interface (called hereafter the reflected rays). Making use of the laws of ray optics, this approximate evaluation should give at least some physical insights into the contribution of partial reflection to radiation pressure.

The procedure is the following. We consider an interface deformation just below the instability threshold. Taking account of the conclusions of Sec. IV E, we suppose that the additional contribution to the radiation pressure of the light partially reflected at the interface is small compared to the radiation pressure due to the incident beam. So, following the conclusions of Sec. VB, we use Eq. (23) as a realistic model of interface shape just below the instability threshold, although Eq. (23) does obviously not contain the mechanism of instability. We determine the direct and reflected rays reaching each point of this interface. We finally compute the associated radiation pressure. This procedure is valid as long as the additional radiation pressure due to the reflected light is small compared to the radiation pressure due to the incident beam (perturbation method).

\section{Selection of rays using ray optics}

Let us consider the point $A_{1}$ of the deformed interface, defined by the radius $r_{1}$, and the height $h_{1}=h\left(r_{1}\right)$. A ray incident on point $A_{2}$ of the interface (of radius $r_{2}$, height $\left.h_{2}=h\left(r_{2}\right)\right)$ is partially reflected towards $A_{1}$ if (i) the following geometrical condition is satisfied [40]:

$$
\frac{h_{1}-h_{2}}{r_{1}-r_{2}}=\tan \left(2 \theta_{2}-\frac{\pi}{2}\right)
$$


where $\theta_{2}=\theta_{i}\left(r_{2}\right)$ is the angle of incidence of the incident ray in $A_{2}, 2 \theta_{2}-\frac{\pi}{2}$ being the angle between the reflected ray and horizontal, and (ii) the ray joining $A_{2}$ to $A_{1}$ propagates in the phase of refractive index $n_{2}$ (see Fig. 19.

Knowing the interface shape $h(r)$, we solve numerically these two conditions at each point $A_{1}$ of the interface. The reflected rays incident on the left side $(r<0)$ of a particular interface profile are plotted in Fig. 20a. A zoom around the tip of the deformation is presented in Fig. 20b. For $r<-3 \mu m$ (area 1 in Fig. 20a), each point $A_{1}$ of the left-hand half-interface is reached by both a direct ray (not represented) and a reflected ray coming from a point $A_{2}$ of the facing, right-hand half-interface $(r>0)$. For $-3 \mu m<r<-0.3 \mu m$ (area 2 in Fig. 20b), each point $A_{1}$ of the left-hand half-interface is reached by a second reflected ray coming from a point $A_{2}$ of the facing half-interface that is very close to the interface tip. For $-0.3 \mu m<r<0$ (area 3 in Fig. 20b), each point $A_{1}$ of the interface is also reached by two additional reflected rays coming from two points $A_{2}$ of the left-hand half-interface (i.e. such that $r_{2}<0$ ). This segmentation of the interface in different areas as a function of the number of reflected rays reaching them evidences the complexity of computing the additional radiation pressure due to reflected light.

\section{Computation of the additional radiation pressure due to reflected light}

After determination of the reflected rays involved in the additional radiation pressure, we are able to compute the radiation pressure associated to these rays. A ray partially reflected at point $A_{2}$ impinges the interface at point $A_{1}$ with an angle of incidence equal to $\pi-2\left|\theta_{2}\right|-\left|\theta_{1}\right|$, where $\theta_{1}=\theta_{i}\left(r_{1}\right)$ is the angle between the horizontal and the interface in $A_{1}$. The intensity $I^{\prime}\left(r_{1}\right)$ of this ray incident at point $A_{1}$ after partial reflection at point $A_{2}$ is

$$
I^{\prime}\left(r_{1}\right)=\frac{r_{2}}{r_{1}} I\left(r_{2}\right) R\left(\theta_{2}\right),
$$

where $I\left(r_{2}\right)$ is the intensity of the direct ray incident at point $A_{2}$ and $R\left(\theta_{2}\right)$ is the energy reflection coefficient of the ray incident at point $A_{2}$. Since $R\left(\theta_{2}\right) \ll 1$ and $I(r)$ rapidly

decreases with $r, I^{\prime}\left(r_{1}\right)$ can be large compared to the intensity $I\left(r_{1}\right)$ of the direct ray only if (i) $\frac{r_{2}}{r_{1}}$ is large, i.e. at points $A_{1}$ close to the beam axis (the divergence at $r_{1}=0$ is an artifact of geometrical optics), or if (ii) $r_{2} \ll r_{1}$, i.e. at high irradiance $I\left(r_{2}\right)$.

Finally, the additional radiation pressure due to reflected light of intensity $I^{\prime}(r)$ is com- 
puted using Eq. (1). In Fig. 21 both the radiation pressure $\Pi$ due to the incident beam of intensity $I(r)$ and the additional radiation pressure $\Pi^{\prime}$ due to the reflected light of intensity $I^{\prime}(r)$ are plotted versus $r$ for the left-hand half-interface used in Fig. 20. Several remarks can be made.

The most important one is that $\Pi^{\prime}$ is found to be much smaller than $\Pi$ for $|r|<10 \mu m$, as assumed by this perturbation method, except very close to the beam axis, where the divergence of $\Pi^{\prime}$ is due to an abusive use of ray optics. This explains why Eq. (23) accurately reproduces the experimental interface shapes, although it does not take into account the additional radiation pressure due to reflected light. Moreover, $\Pi$ ' is found to be much larger than $\Pi$ for $|r|>10 \mu m$ because $\left|r_{2}\right| \ll\left|r_{1}\right|$, i.e. $I\left(r_{2}\right) \gg I\left(r_{1}\right)$. However, for $|r|>10 \mu m$ the total radiation pressure is very small (less than $10^{-5} \mathrm{~Pa}$ ).

Secondly, the steep variations of $\Pi^{\prime}(r)$ are due either to the total reflection at point $A_{1}$ of the reflected ray, or to the contribution of an additional reflected ray as $|r|$ decreases, corresponding to the transition from area 1 (resp. 2) to area 2 (resp. 3), as described in section B 1 .

The next step of this procedure would be to compute the interface shape resulting from the radiation pressure of both direct and reflected rays, and to iterate the computation of the radiation pressure field (procedure of the Hartree-Fock type) in order to investigate whether it leads to instability. The nonrealistic divergence of the light intensity at the hump tip nonetheless makes this iteration quantitatively non pertinent.

To summarize this appendix, we showed using ray optics that, close to the beam axis, the additional radiation pressure due to the light partially reflected on the interface is small compared to the radiation pressure due to the incident laser beam.

[1] J. C. Maxwell, A Treatise on Electricity and Magnetism, Reprinted by Dover Books, New York, 1954.

[2] R. Loudon, Fortschr. Phys. 52, 1134 (2004).

[3] M. Mansuripur, Optics Express 12, 5375 (2004).

[4] A. Feigel, Phys. Rev. Lett. 92, 020404 (2004). R. Schützhold and G. Plunien, Phys. Rev. Lett. 93, 268901 (2004). A. Feigel, Phys. Rev. Lett. 93, 268902 (2004). B. A. van Tiggelen and G. 
L. J. A. Rikken, Phys. Rev. Lett. 93, 268903 (2004). A. Feigel, Phys. Rev. Lett. 93, 268904 (2004).

[5] P. Lebedev, Ann. Phys. (Liepzig) 6, 433 (1901).

[6] E. F. Nichols and G. F. Hull, Phys. Rev. 17, 26 (1903).

[7] J. H. Poynting, Philos. Mag. 9, 393 (1905). J. H. Poynting, The Pressure of Light (Society for the propagation of the Christian knowledge, London, 1910).

[8] J. J. Thomson, Electricity and Magnetism (Constable, London, 1904).

[9] A. Ashkin and J. M. Dziedzic, Phys. Rev. Lett. 30, 139 (1973).

[10] I. Brevik, Phys. Rep. 52, 133 (1979).

[11] R. Loudon, J. Mod. Optics 49, 821 (2002).

[12] Y. N. Obukhov and F. W. Hehl, Phys. Lett. A 311, 277 (2003).

[13] J. P. Gordon, Phys. Rev. A 8, 14 (1973).

[14] G. V. Ostrovskaya, I. I. Komissarova, and E. N. Shedova, Optics Comm. 66, 15 (1987).

[15] J. Z. Zhang and R. K. Chang, Opt. Lett. 13, 916 (1988)

[16] K. Sakai, D. Mizuno, and K. Takagi, Phys. Rev. E 63, 046302 (2001)

[17] K. Sakai, K. Tachibana, S. Mitani, and K. Takagi, J. Colloid Interface Sci. 264, 446 (2003).

[18] A. Casner and J.P. Delville, Phys. Rev. Lett. 87, 054503 (2001).

[19] S. Mitani and K. Sakai, Phys. Rev. E 66, 031604 (2002).

[20] J. P. Gordon, R. C. C. Leite, R. S. Moore, S. P. S. Porto, and J. R. Whinnery, J. Appl. Phys. 36, 3 (1965).

[21] J. P. Longtin, K. Hijikata, and K. Ogawa, Int. J. Heat Mass Transfer 42, 85 (1999).

[22] A. Ashkin, Proc. Natl. Acad. Sci. USA 94, 4853 (1997).

[23] M. I. Angelova, B. Pouligny, G. Martinot-Lagarde, G. Gréhan, G. Gouesbet, Progr. Colloid. Polym. Sci. 97, 293 (1994)

[24] A. Richert, S. Hénon, G. Le Normand, and F. Gallet, Biophys. J. 76, 1145 (1999).

[25] C. L. Guo, C. H. Lee, and J. Wang, Opt. Lett. 23, 307 (1998).

[26] J. Guck, R. Ananthakrishnan, T. J. Moon, C. C. Cunningham and J. Käs, Phys. Rev. Lett. 84, 5451 (2000). Biophys. J. 81767 (2001).

[27] W. C. Lin, C. H. Lee, and J. Wang, Phys. Rev. E 64, 020901 (2001).

[28] H. M. Lai, P. T. Leung, K. L. Poon, and K. Young, J. Opt. Soc. Am. B 6, 2430 (1989).

[29] I. Brevik and R. Kluge, J. Opt. Soc. Am. B 16, 976 (1999). 
[30] A. Casner and J. P. Delville, Phys. Rev. Lett. 90, 144503 (2003).

[31] R. Wunenburger, A. Casner, and J.P. Delville, submitted to Phys. Rev. E.

[32] M. Born and E. Wolf, Principles of Optics, $6^{\text {th }}$ Ed. (Pergamon, Oxford, 1980).

[33] A. Casner, J.P. Delville, I. Brevik, J. Opt. Soc. Am. B 20, 2355 (2003).

[34] J. Meunier, A. M. Cazabat, D. Langevin, and A. Pouchelon, J. Physique Lett. 43, L-89 (1982).

[35] E. Freysz, Ph.D. thesis, Bordeaux I University, 1990.

[36] B. Jean-Jean, E. Freysz, A. Ducasse, and B. Pouligny, Europhys. Lett. 7, 219 (1986).

[37] A. Casner and J.P. Delville, Opt. Lett. 26, 1418 (2001).

[38] X. Chavanne, Ph.D. thesis, Joseph Fourier University, 1997. A. B. Kogan, D. Murphy, and H. Meyer, Phys. Rev. Lett. 82, 4635 (1999).

[39] H. M. Lai, Phys. Rev. Lett. 92, 049401 (2004).

[40] M. D. Betterton, Phys. Rev. E 63, 056129 (2001). 


\section{Captions}

Figure 1: Variation of photon momentum (solid arrows) at the interface between two dielectric, non magnetic liquids. (a) downward propagating photon. (b) upward propagating photon. Dotted arrows represent the momentum transferred from the photon to the interface. $\rho_{1}$ and $\rho_{2}$ are the densties of the two fluids and $\vec{g}$ is the acceleration of gravity.

Figure 2: Picture of the steady interface just below the instability threshold in order to illustrate the notations. The interface is deformed by a laser beam of power $P=480 \mathrm{~mW}$ and waist $\omega_{0}=3.47 \mu m$, propagating downward. The sample temperature $T$ is $41^{\circ} C$, i.e. $6 K$ above the critical temperature $T_{c}$. Incidence, reflection and transmission are illustrated by the corresponding arrows. $\theta_{i}$ and $\theta_{t}$ are respectively the incident and the refracted angles.

Figure 3: left: schematic phase diagram of the micellar phase of micro-emulsion for the composition described in text. $\phi$ is the micelle volume fraction, $T$ is temperature. Right: corresponding representation of the phase distribution within the sample in a two-phase state $\left(T>T_{c}\right)$.

Figure 4: experimental setup.

Figure 5: (a) Pictures of interface deformations due to the radiation pressure of a laser beam propagating downward of waist $\omega_{0}=7.4 \mu \mathrm{m}$ at $T-T_{c}=3 \mathrm{~K}$. Bottom: the digitized interface shapes (o) of the pictures above are compared to the predicted shapes (solid lines) computed using Eq. (12). (b) Same as (a) for an laser beam propagating upward of waist $\omega_{0}=14.6 \mu m$ at $T-T_{c}=2 K$.

Figure 6: Variations of $h(r=0)$ versus $\frac{P\left(n_{2}-n_{1}\right)}{\sigma c}$ in the $B o \ll 1$ regime. Measurements are performed at $T-T_{c}=8,10,15,20$, and $25 K$ for each beam waist value. The solid line is the best linear fit.

Figure 7: Variations of $h(r=0)$ versus $\frac{n_{2}-n_{1}}{\rho_{2}-\rho_{1}} \frac{P}{g c \omega_{0}^{2}}$ for $B o>2$. Measurements are performed at $\omega_{0}=25.3,29.3$ and $32.1 \mu m$ for each $T-T_{c}$ value. The solid line is the best linear fit.

Figure 8: Variations of the normalized hump height $\frac{h(r=0)}{h(r=0)_{B o \gg 1}}$ versus the $B o$ number measured for both upward (o) and downward (•) laser beams. The solid line corresponds to the function $F(B o)$ given by Eq. (16).

Figure 9: Variation of the hump height $h(r=0)$ versus the beam power $P$ when light propagates upward $(\mathbf{\Delta})$ and downward $(\nabla)$ for $\omega_{0}=5.3 \mu m$ and $T-T_{c}=3 K$. Right-hand picture: tether-like shape of the interface deformation appearing for increasing $P$ when light 
propagates upward. Left-hand picture: long jet observed above a beam power threshold $P_{\uparrow}$, illustrated by the dash-dotted line, when light propagates downward.

Figure 10: (a) Variation of the beam power threshold $P_{\uparrow}$ versus the beam waist $\omega_{0}$ for several values of $T-T_{c}$. Lines represent linear fits. (b) Variation of $P_{\uparrow}$ versus $T-T_{c}$ for $\omega_{0}=3.5 \mu \mathrm{m}$ in log-log scales. The solid line is a power-law fit $P_{\uparrow} \sim\left(T-T_{c}\right)^{1.01 \pm 0.05}$.

Figure 11: Rescaling of the data set presented in Fig. 10a according to the scaling law given by Eq. (19). The solid line is the best linear fit.

Figure 12: Proposed mechanism at the origin of the interface instability and the jet formation at $P_{\uparrow}$ showing optical lensing $(t=1.2 s)$ followed by total reflection of light at the edge of the deformation $(t=2.4 s)$ and the resulting optical guiding by the induced jet $(t=3.6 \mathrm{~s}$ and $t=7.2 \mathrm{~s})$. The experiment is performed at $T-T_{c}=5 \mathrm{~K}, \omega_{0}=3.5 \mu \mathrm{m}$ and $P=P_{\uparrow}=460 \mathrm{~mW}$.

Figure 13: Digitization of the interface shape shown in Fig. 2. $(+)$ : digitized left and right sides of the interface shape $r_{l}(h)$ and $r_{r}(h)$. (o) : tip digitization. Solid lines are fits of $r_{l}(h)$ and $r_{r}(h)$ by polynomial functions of third degree $Q_{l}(h)$ and $Q_{r}(h)$.

Figure 14: Variation of the maximum angle of incidence $\theta_{i \max }$ along the interface measured just below the instability onset versus $T-T_{c}$. Solid line: Variation of the angle of total reflection $\theta_{T R}$ predicted by Eq. (20).

Figure 15: Variation of the maximum angle of incidence $\theta_{i \max }$ along the interface measured just below the instability onset versus $\omega_{0}$. The lines decorated with open symbols represent the corresponding values of the angle of total reflection $\theta_{T R}$ predicted by Eq. (20).

Figure 16: (a) Energy reflection coefficient $R$ of a TE wave at the interface as a function of its angle of incidence $\theta_{i}$, for several values of $T-T_{c}$. Symbols are a guide for the eye. (b) Energy reflection coefficient growth rate $\frac{1}{R} \frac{d R}{d \theta_{i}}$ as a function of the angle of incidence $\theta_{i}$. Symbols are the same as (a).

Figure 17: Experimental interface profiles at $T-T_{c}=8 \mathrm{~K}$ just below the instability onset (see Fig. 13 for symbols) represented in reduced coordinates, for several values of the beam waist. Solid lines : numerical interface profiles computed from Eq. 23 so that $\theta_{i \max }=72^{\circ}$.

Figure 18: Schematics used for the calculation of the radiation force exerted by a laser beam on an interface separating two dielectric fluids characterized by the refractive indices $n_{i}$ and $n_{t} . \theta_{i}$ and $\theta_{t}$ are respectively the incidence and the refracted angles. $\mathbf{t}$ and $\mathbf{n}$ are the tangent and the normal directions at the location where the beam impinges the interface. 
Figure 19: Geometry of the radiation pressure contribution of light partially reflected at the interface. $A_{1}$ is reached by a direct incident ray and a ray having been previously reflected in $A_{2}$.

Figure 20: Shape of an interface at $T-T_{c}=10 K$ deformed by a $\omega_{0}=3.47 \mu m$, $P=822 \mathrm{~mW}$ laser beam, computed using Eq. (22). The light rays reaching regularly spaced points of the left-hand half interface after one reflection are represented. (a) wide view of the interface. 1 labels the family of points of the interface reached by only one reflected ray. (b) enlarged view of the interface tip. 2 (resp. 3) labels the family of points of the interface reached by 2 reflected rays (resp. 4).

Figure 21: Radiation pressure contributions acting on the interface shown in Fig. 20. $\Pi$ is the contribution of the direct incident light, whereas $\Pi^{\prime}$ is the contribution of the light having been previously reflected one time at the interface. 

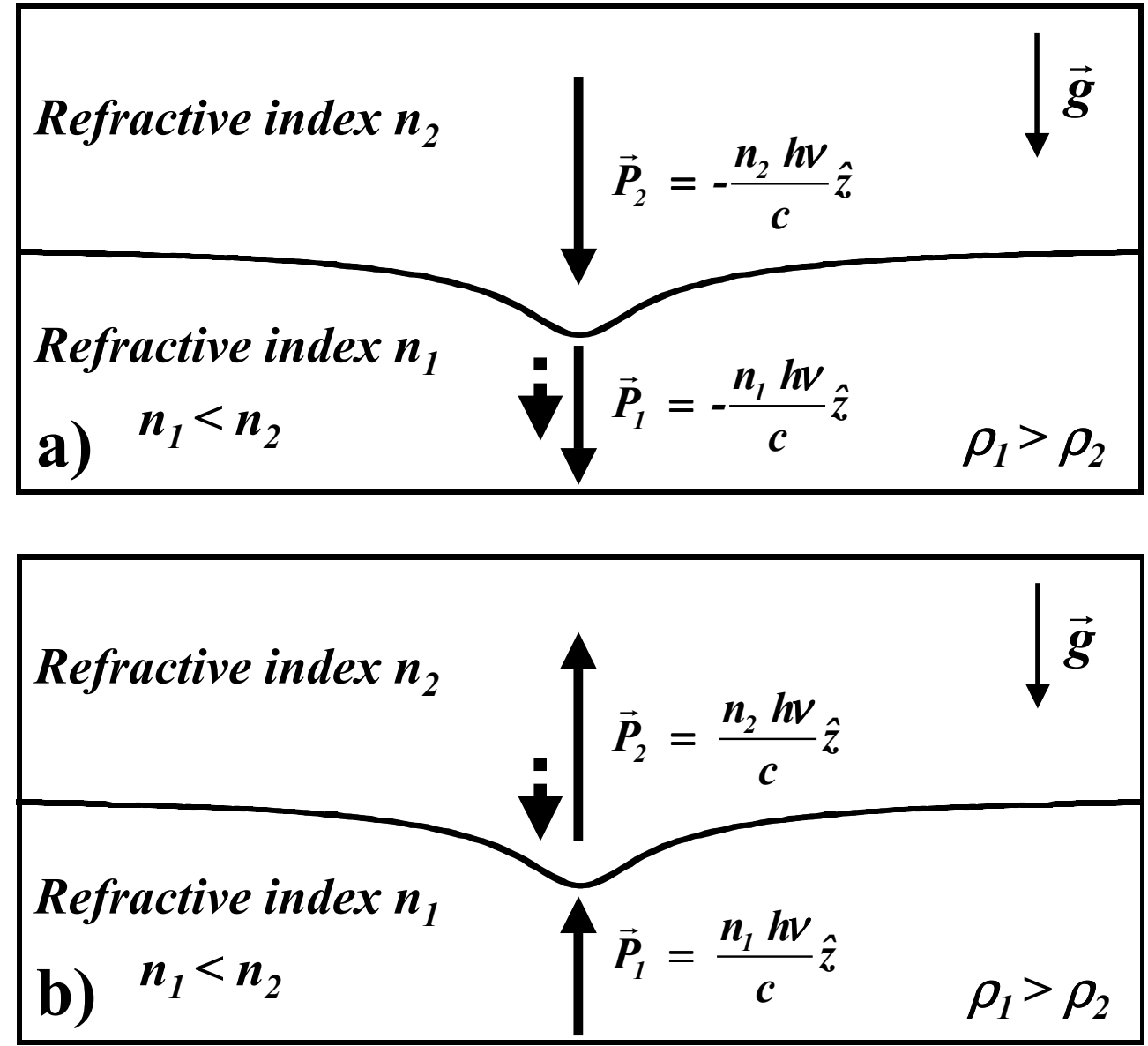

FIG. 1: 


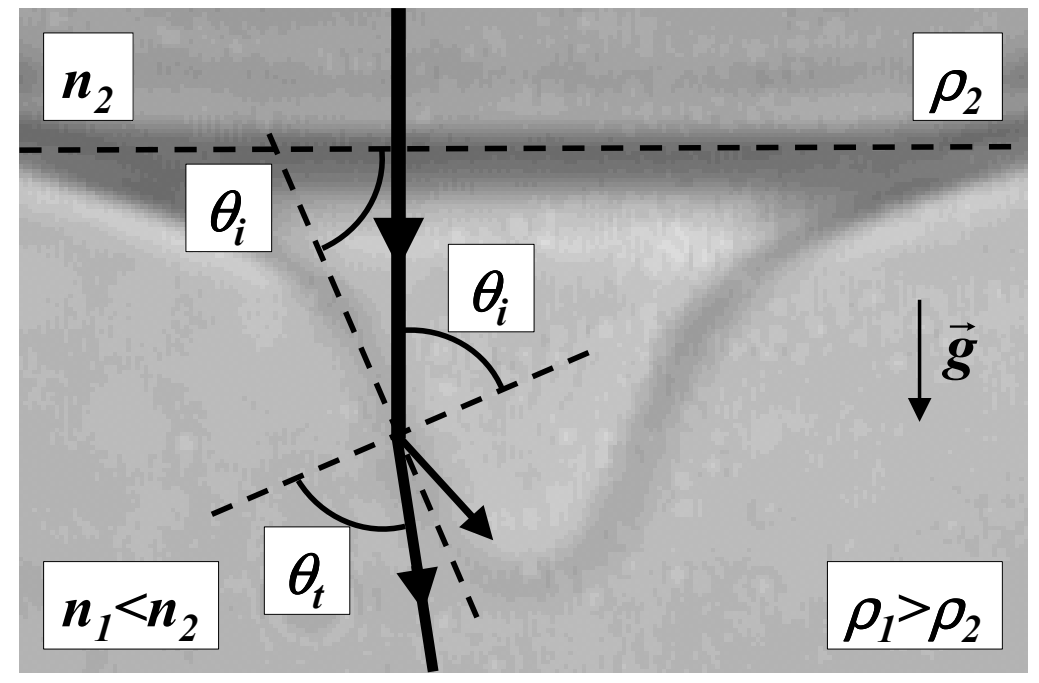

FIG. 2: 


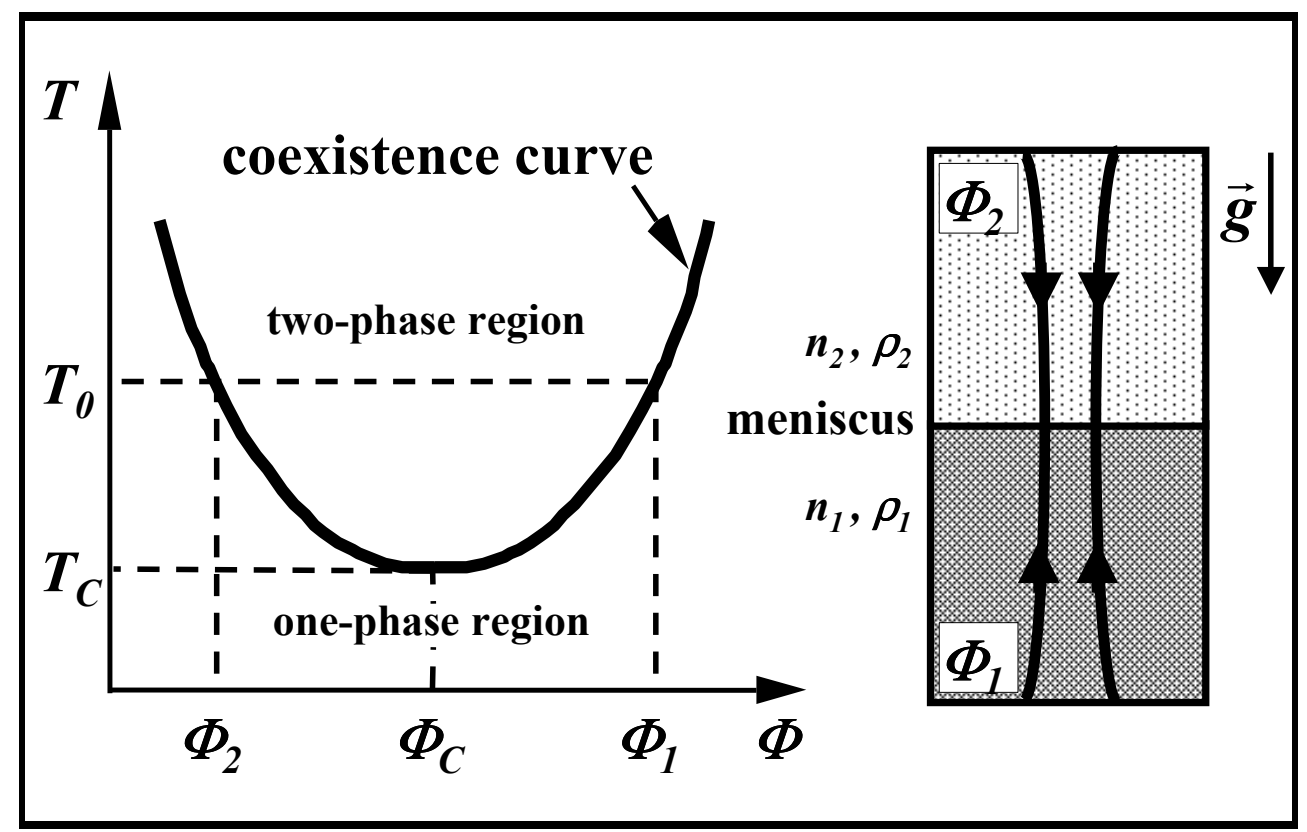

FIG. 3: 


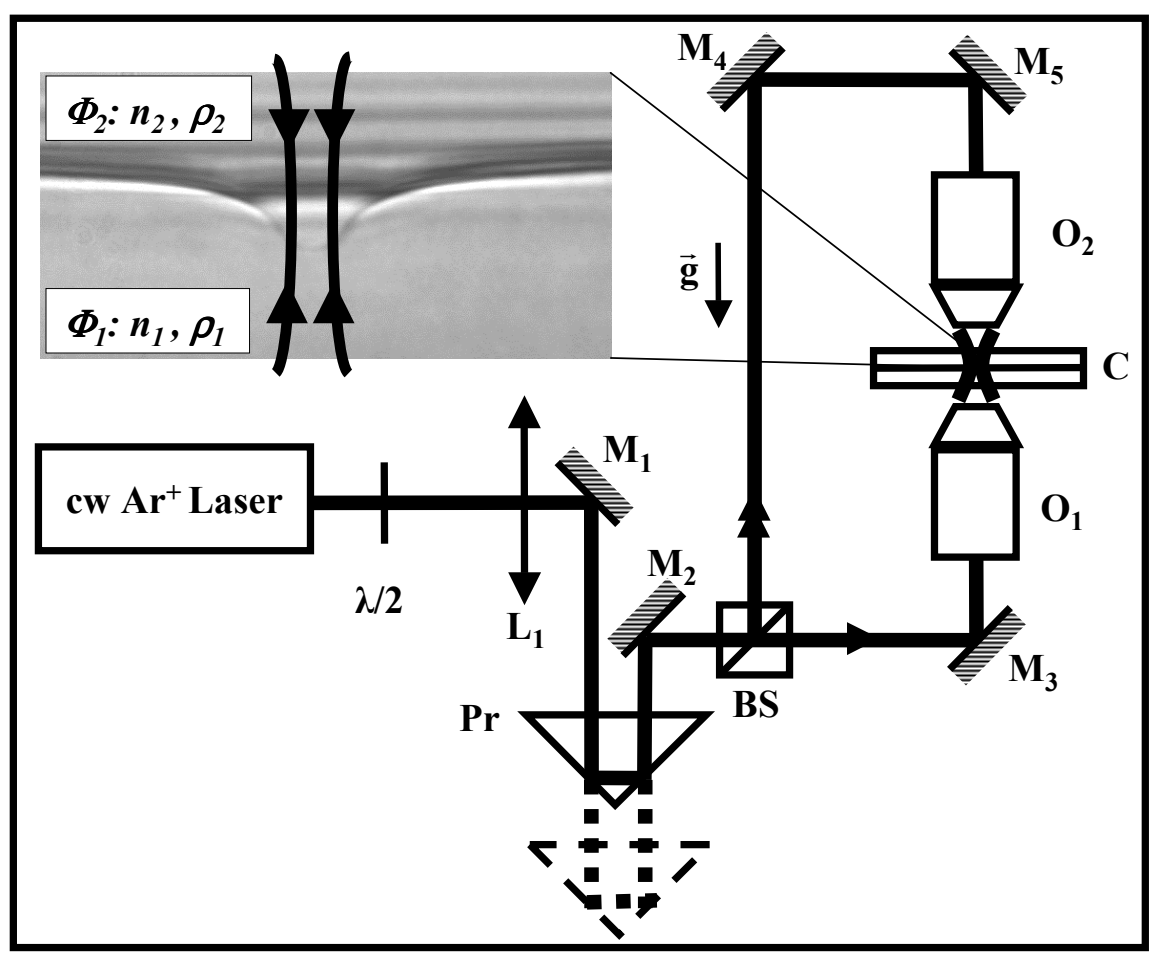

FIG. 4: 

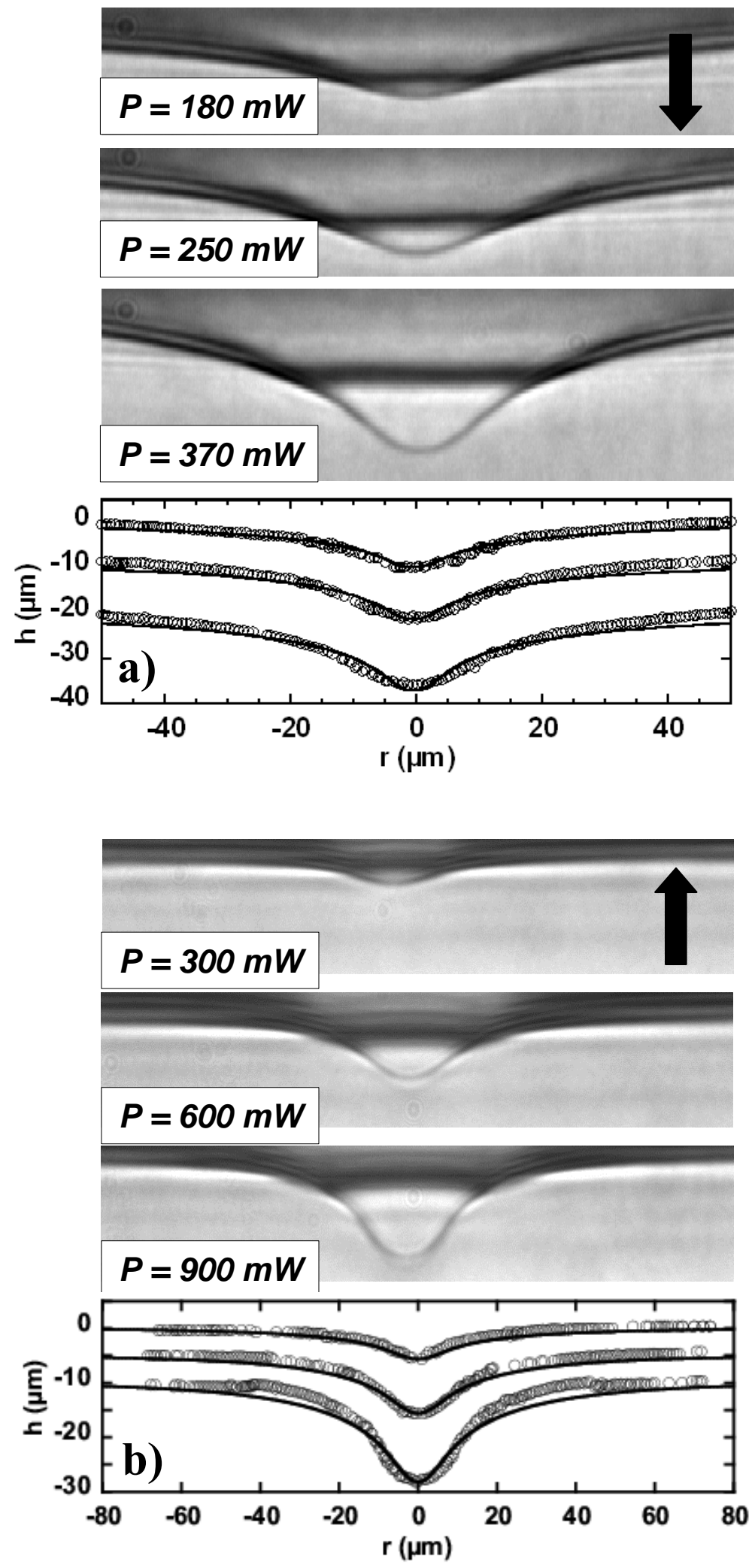


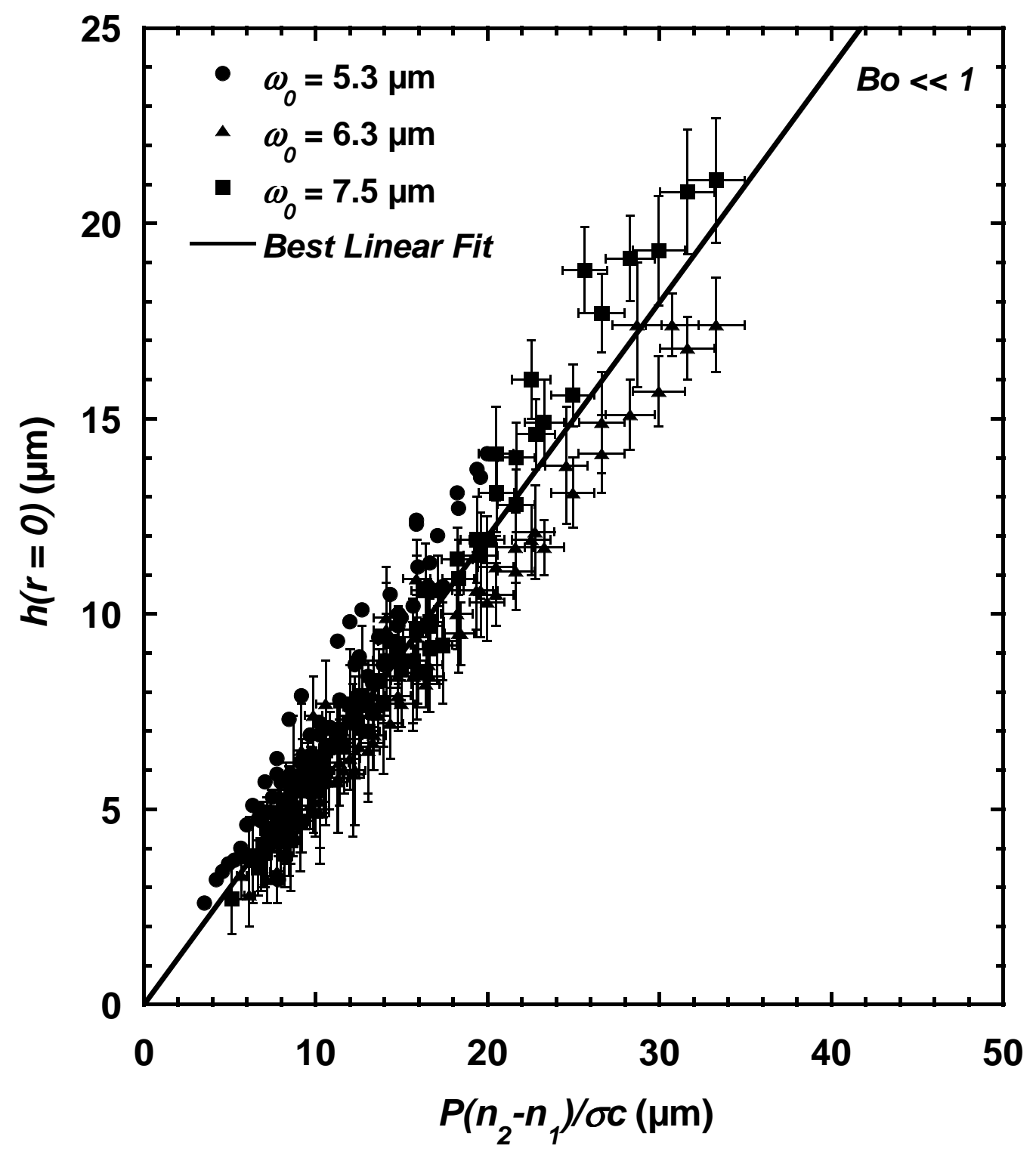

FIG. 6: 


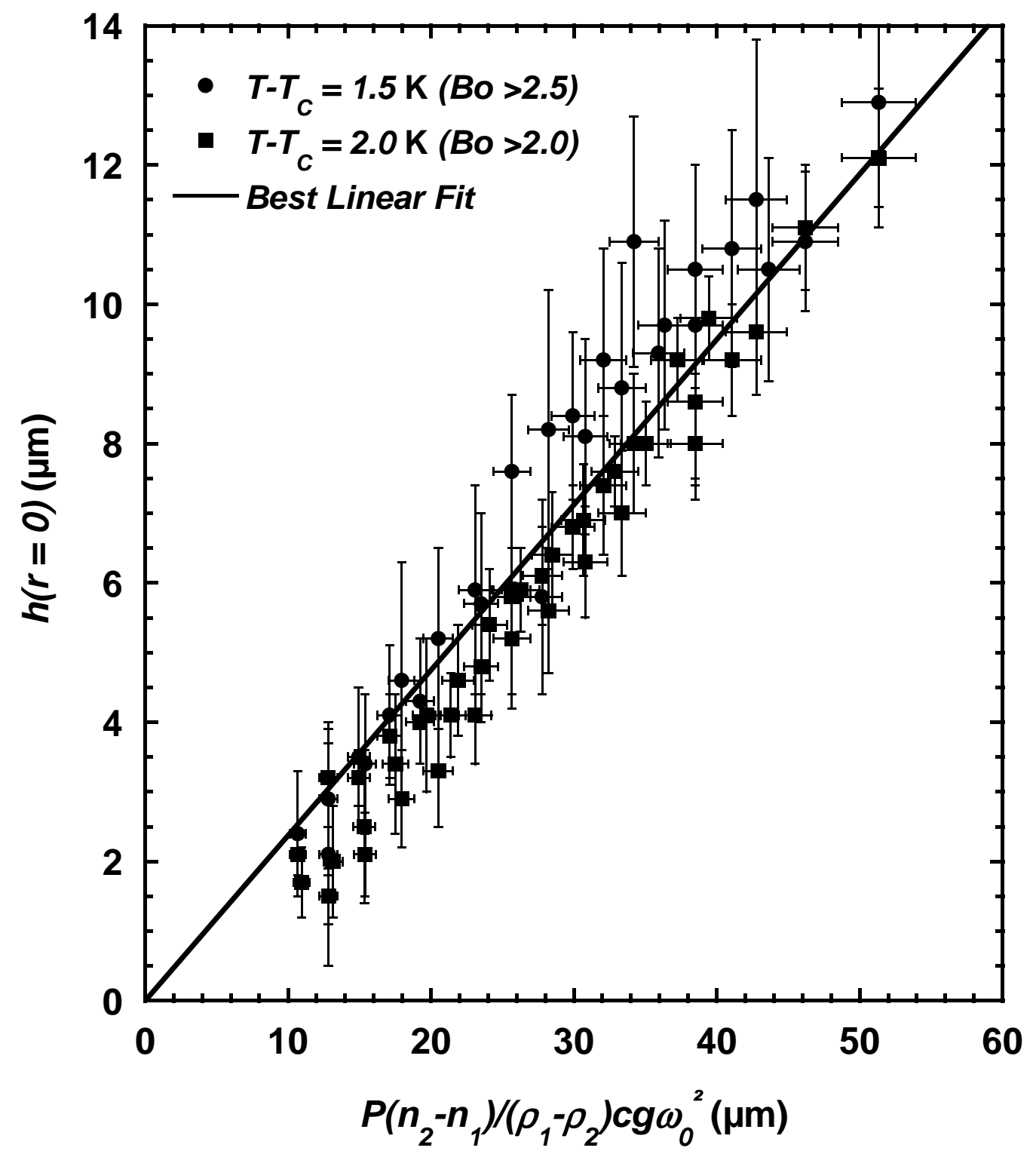

FIG. 7: 


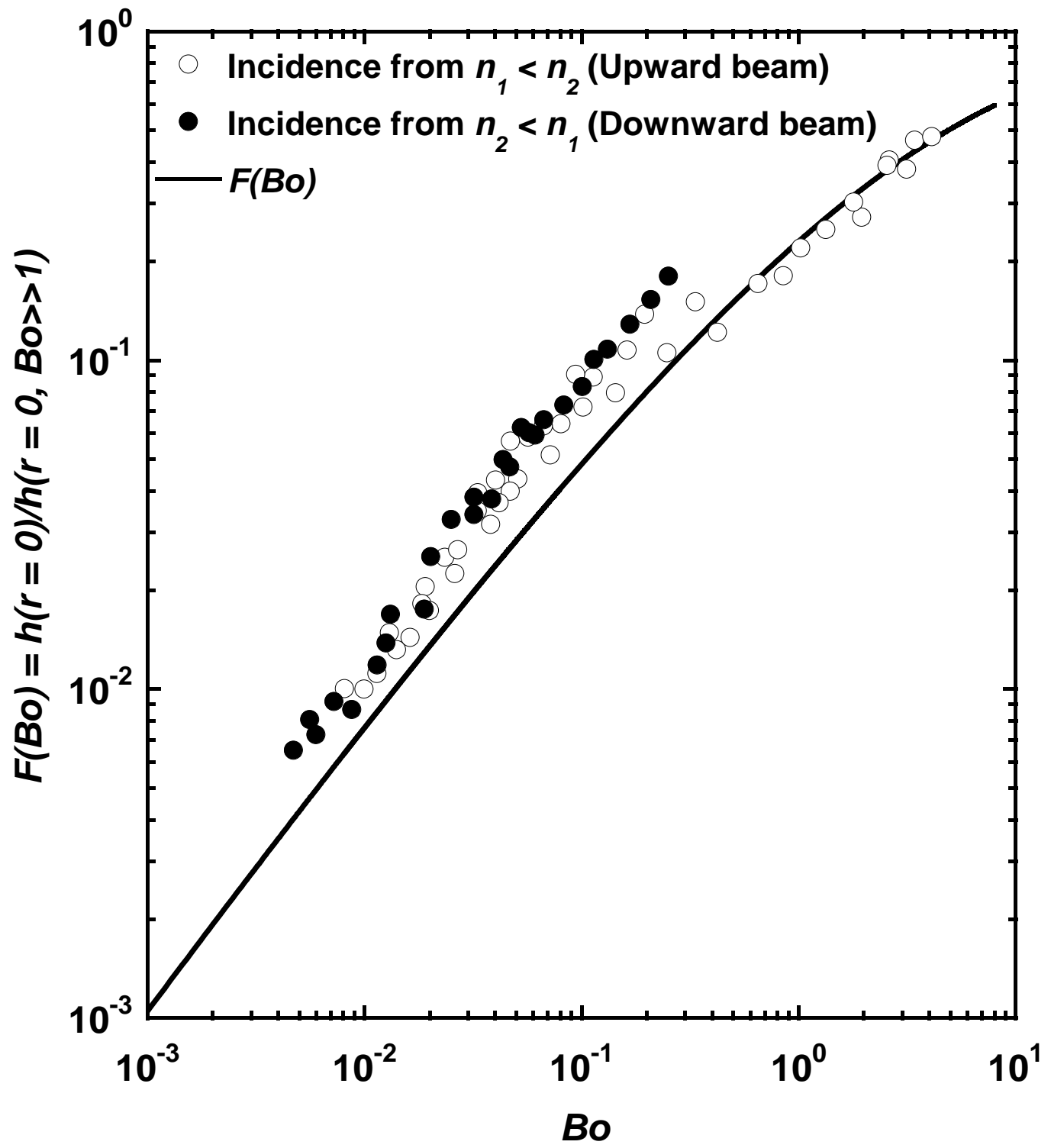

FIG. 8: 


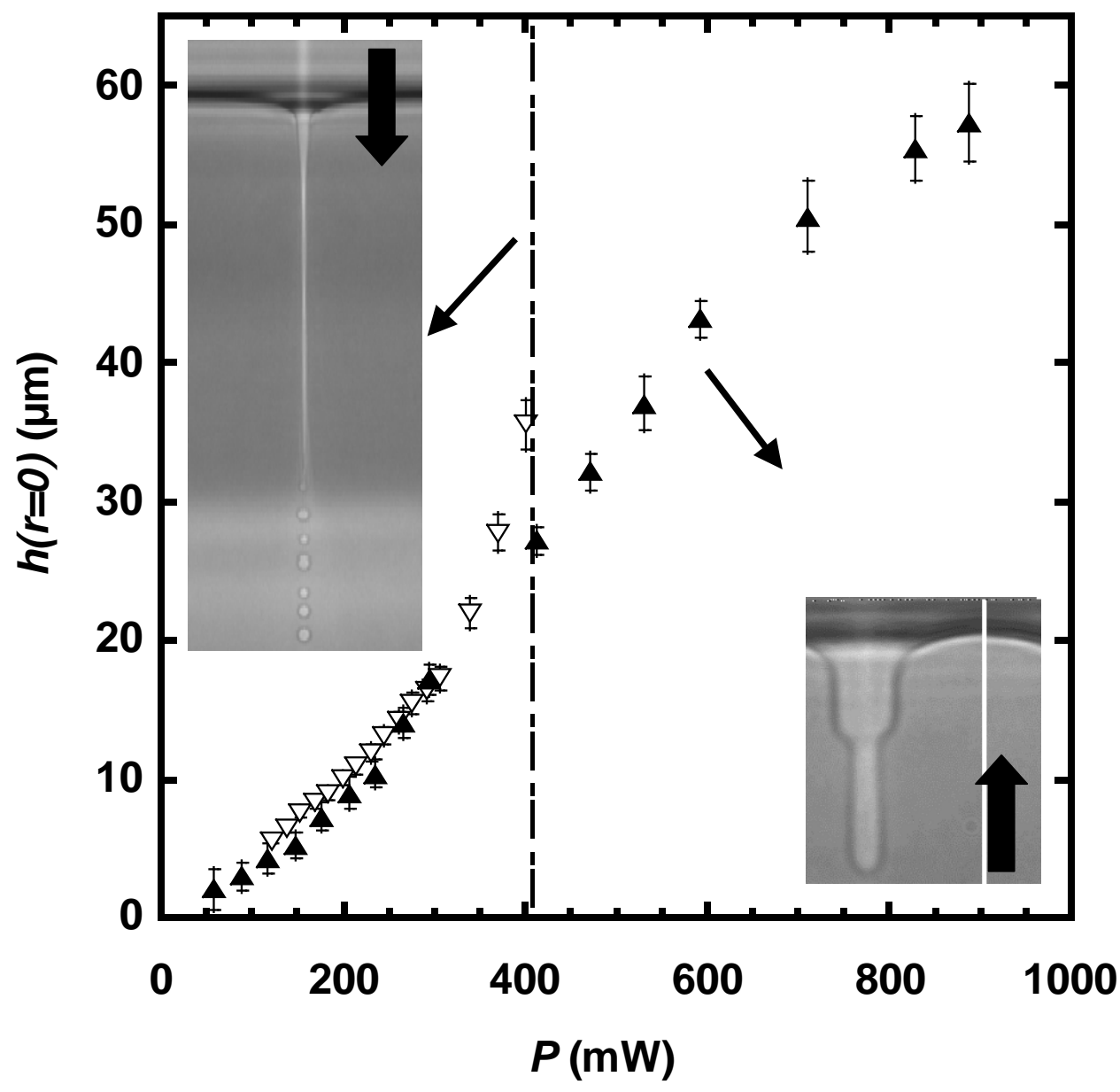

FIG. 9: 

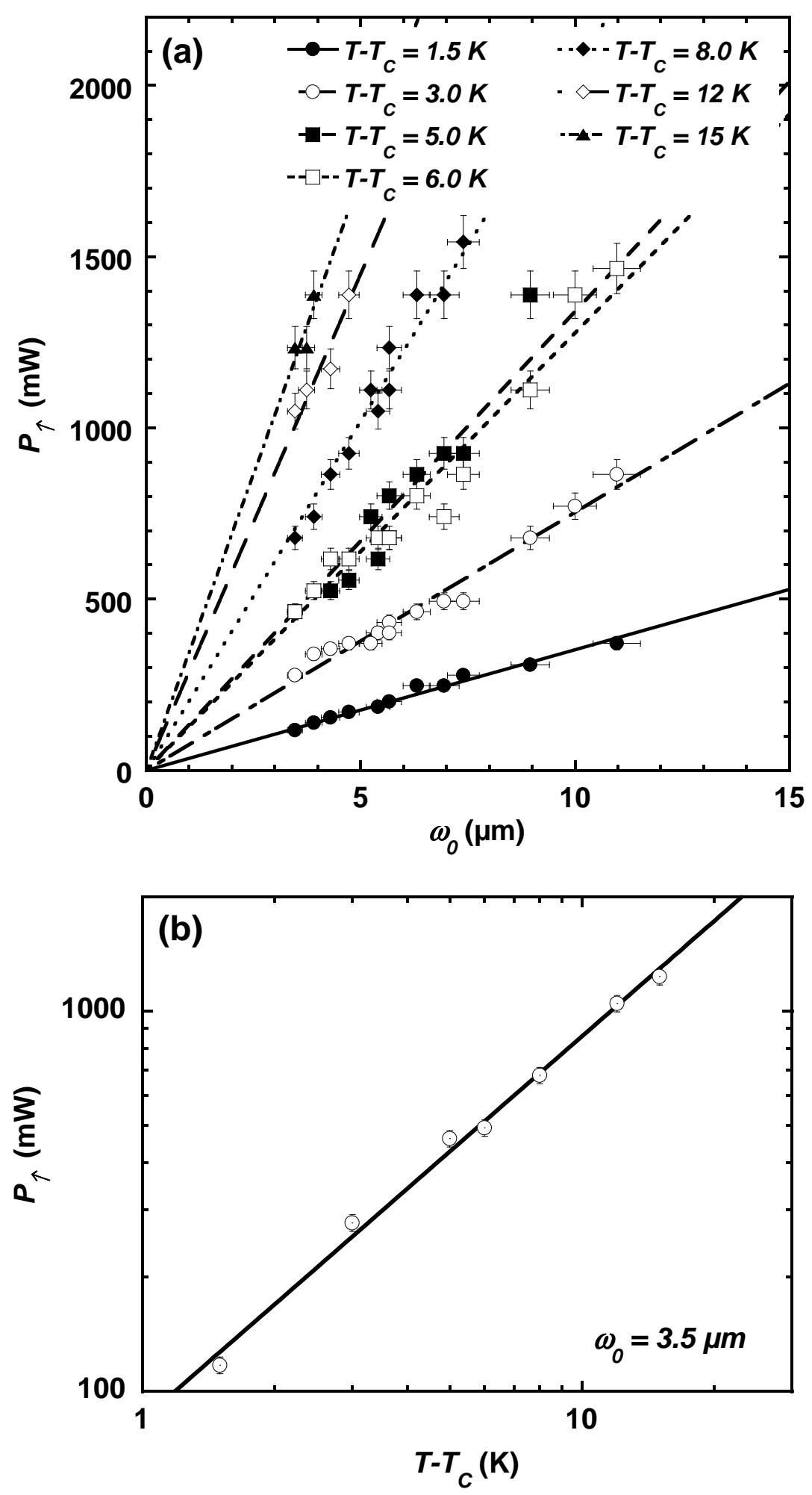


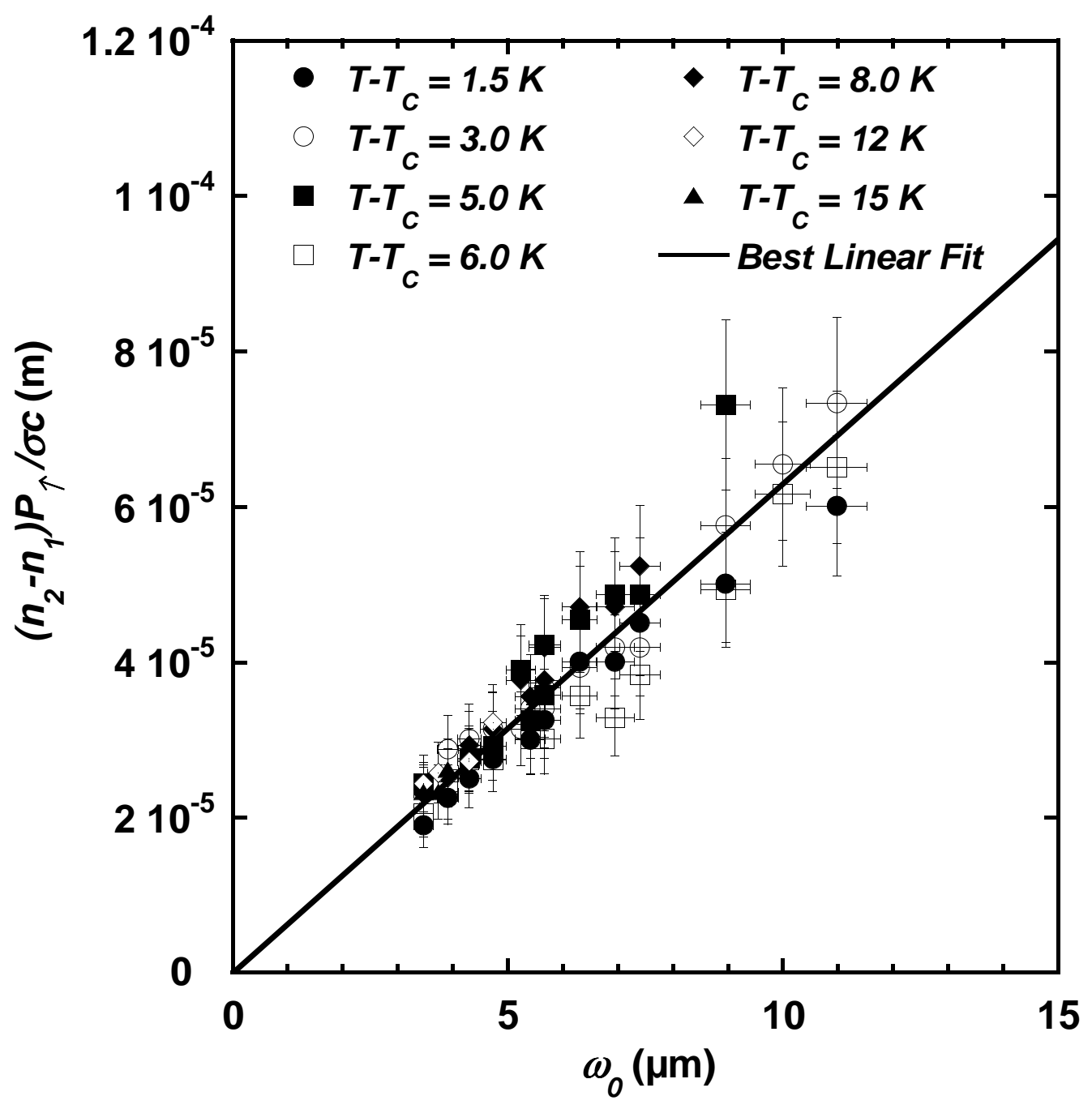

FIG. 11: 


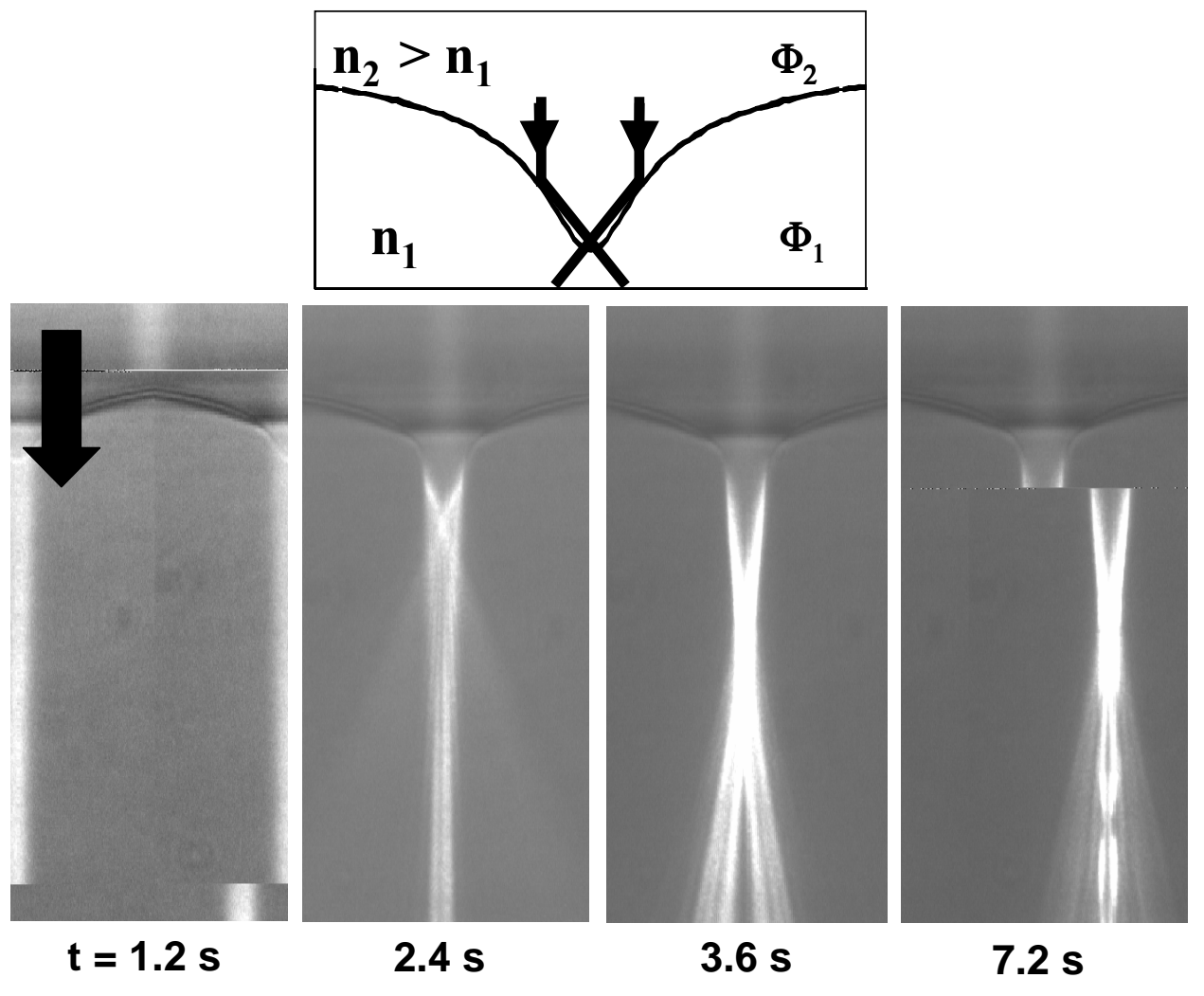

FIG. 12: 


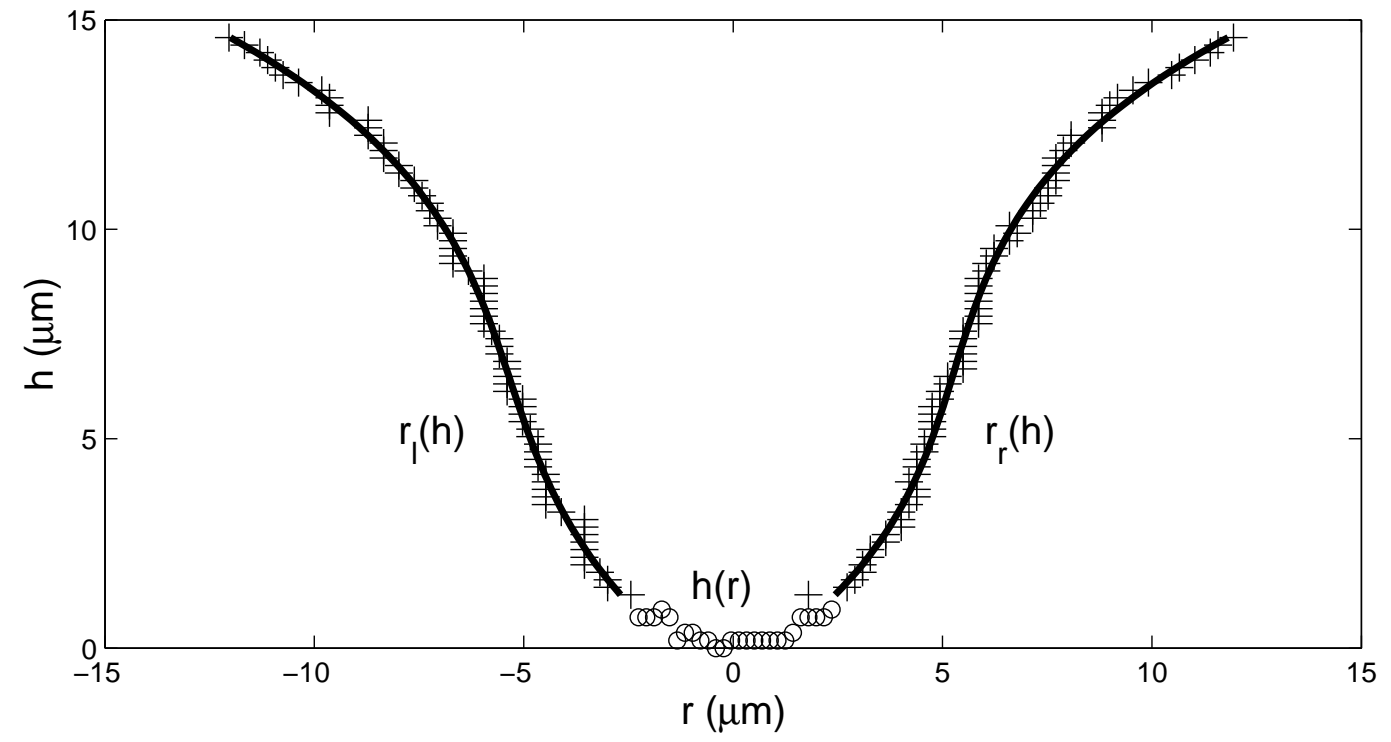

FIG. 13: 


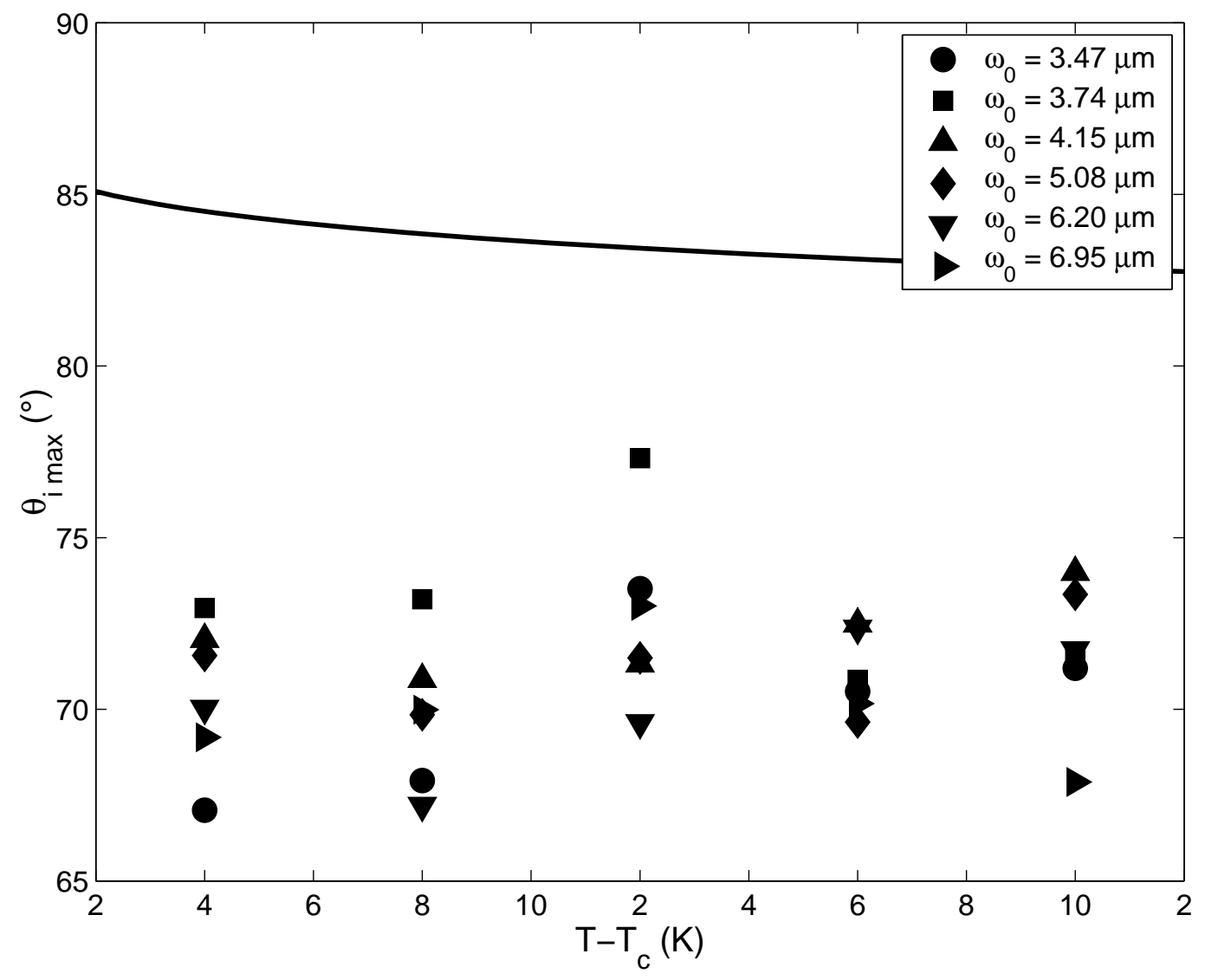

FIG. 14: 


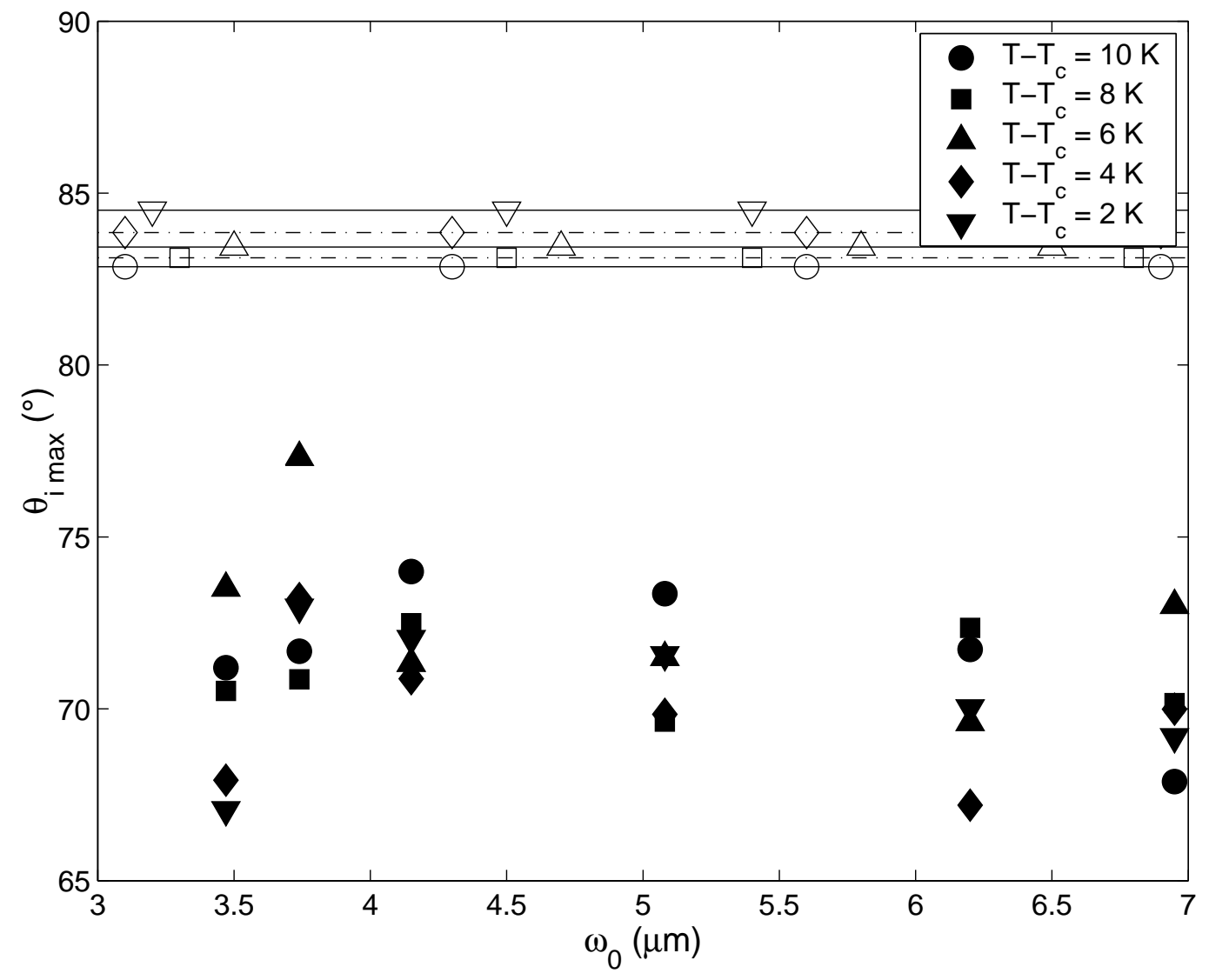

FIG. 15: 

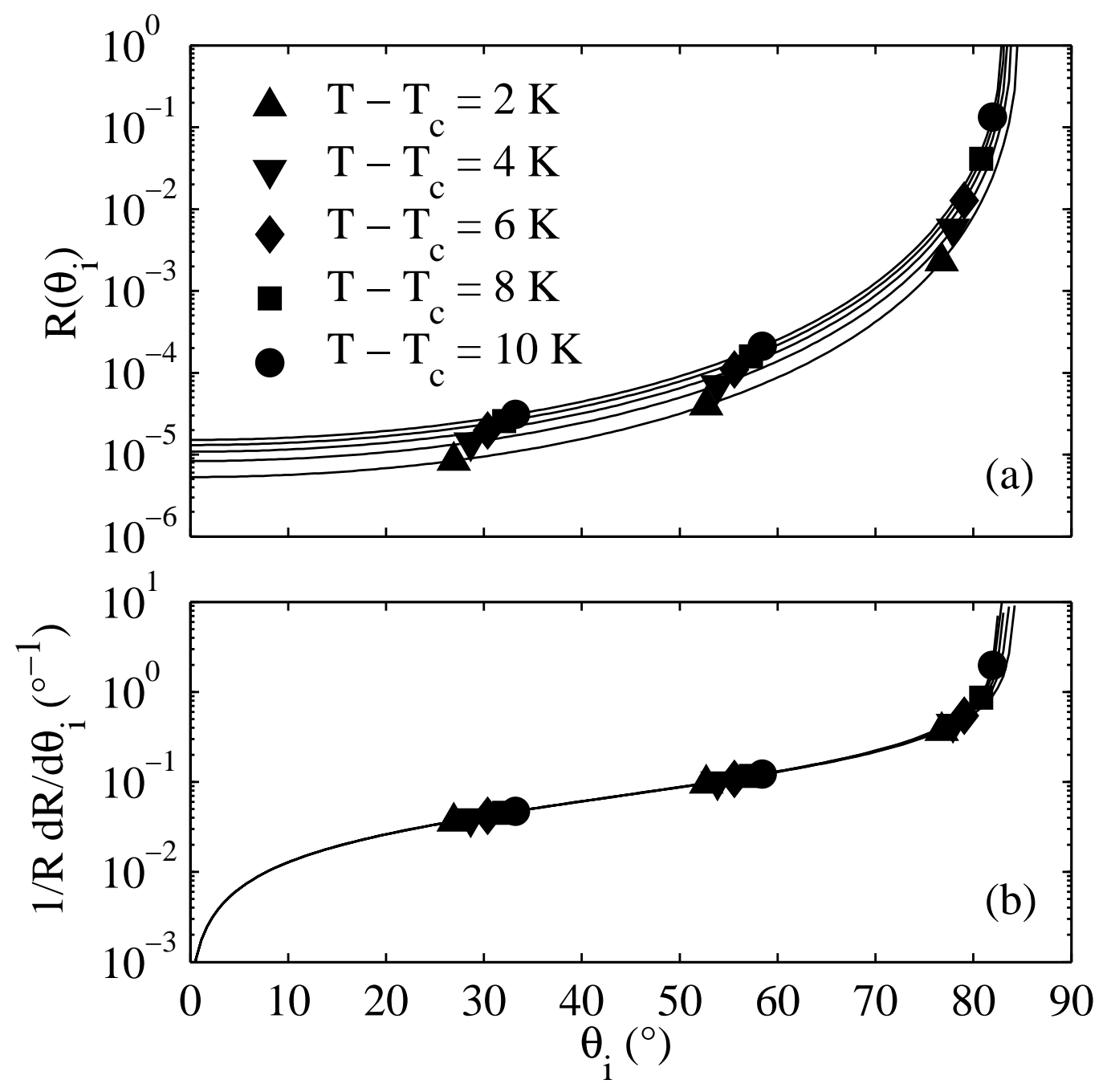

FIG. 16: 

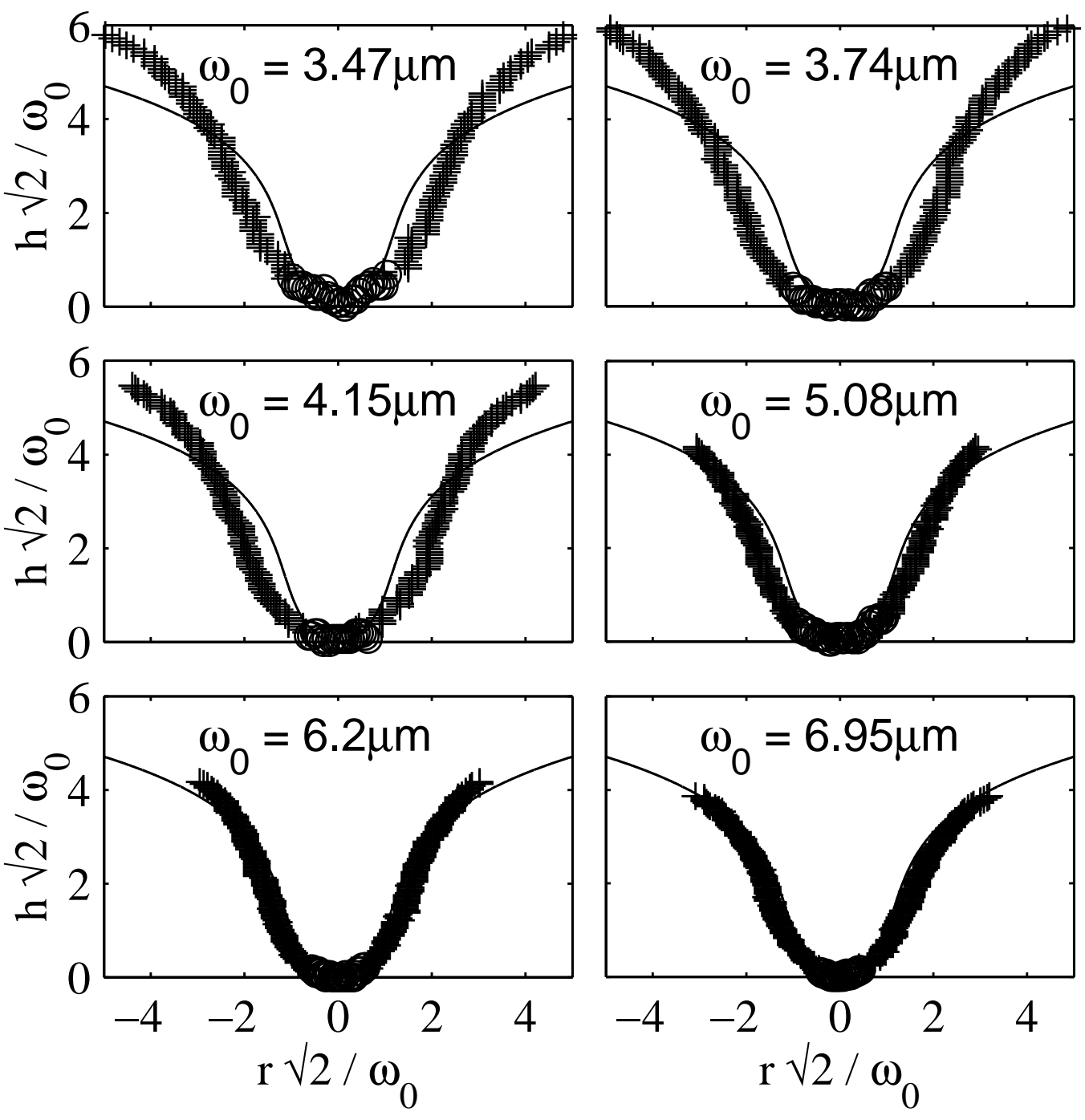

FIG. 17: 


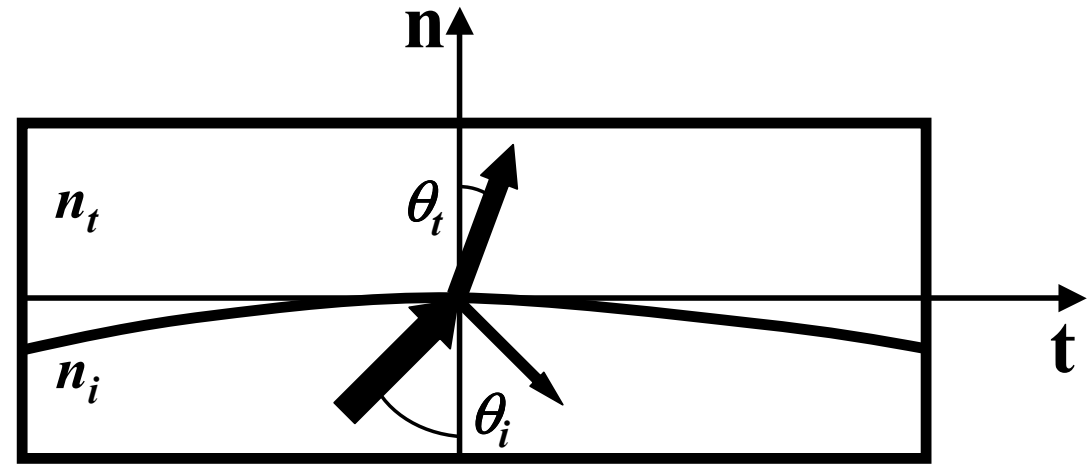

FIG. 18: 


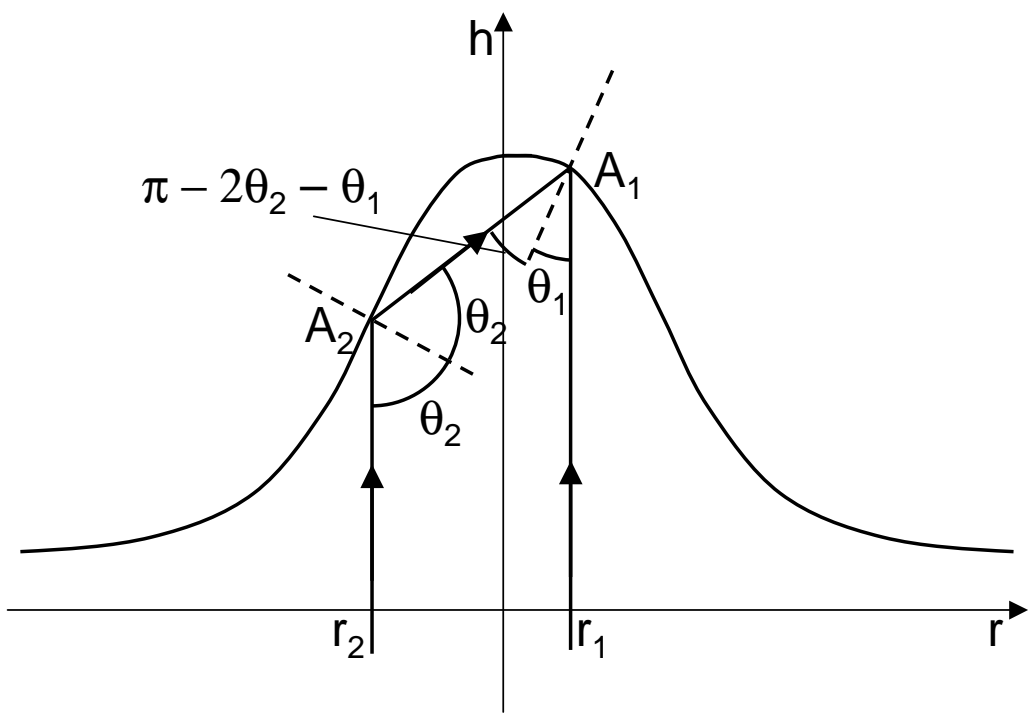

FIG. 19: 

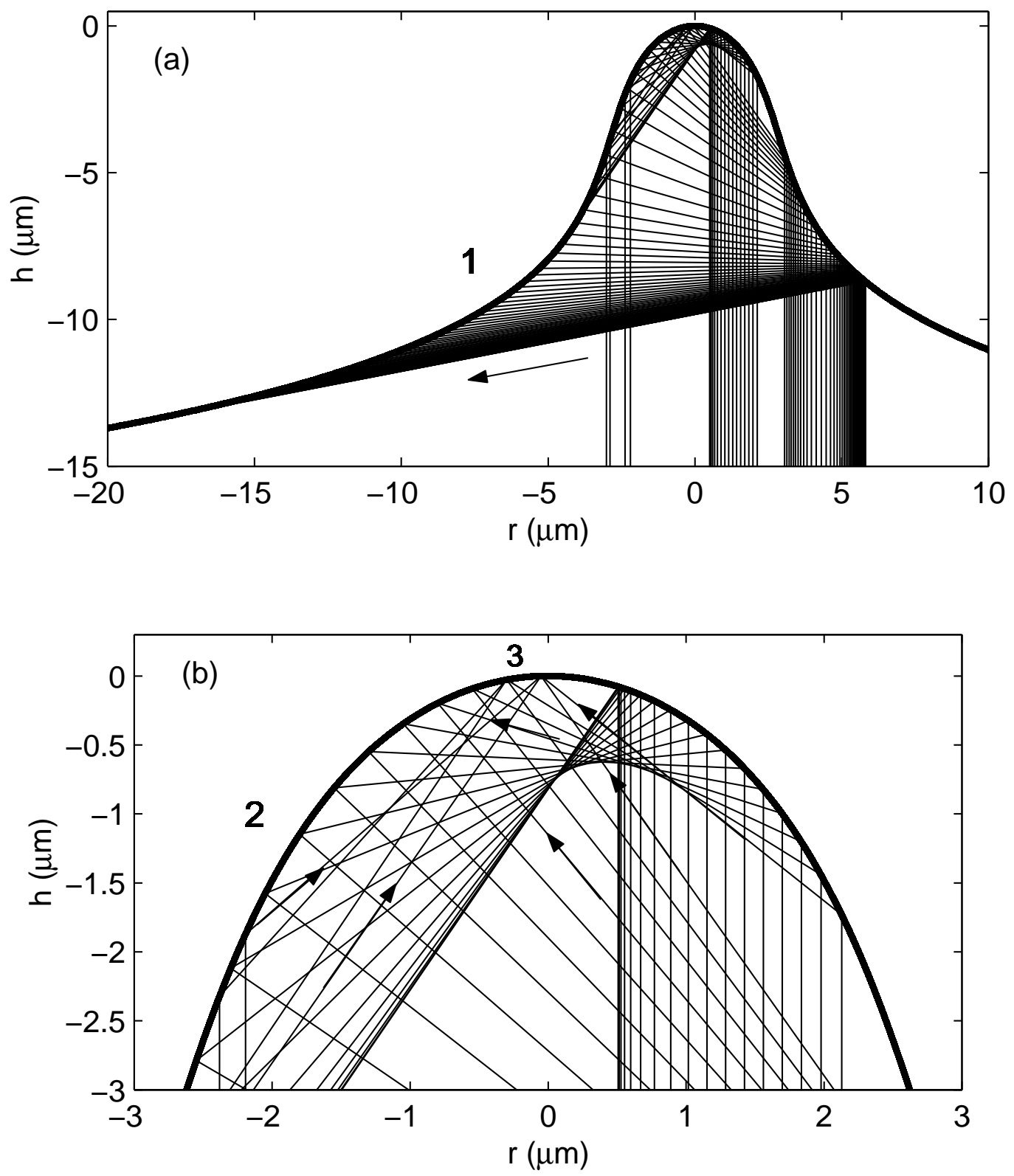

FIG. 20: 


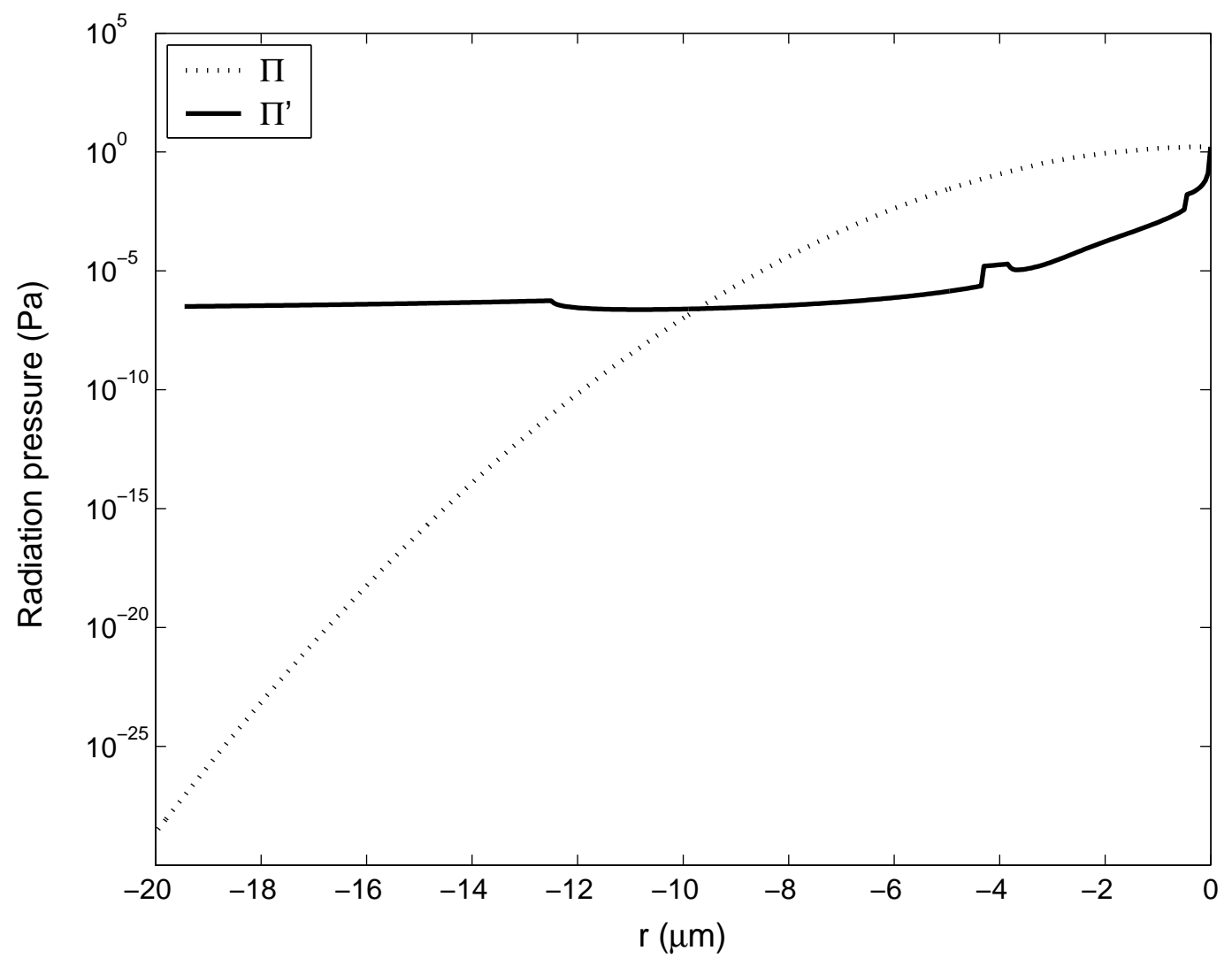

FIG. 21: 